\title{
Semigroups with finitely generated universal left congruence
}

\author{
Yang Dandan ${ }^{1}$. Victoria Gould ${ }^{2}$ D . Thomas Quinn-Gregson ${ }^{3}$. \\ Rida-E Zenab ${ }^{2,4}$
}

Received: 10 December 2018 / Accepted: 4 February 2019 / Published online: 19 February 2019

(c) The Author(s) 2019

\begin{abstract}
We consider semigroups such that the universal left congruence $\omega^{\ell}$ is finitely generated. Certainly a left noetherian semigroup, that is, one in which all left congruences are finitely generated, satisfies our condition. In the case of a monoid the condition that $\omega^{\ell}$ is finitely generated is equivalent to a number of pre-existing notions. In particular, a monoid satisfies the homological finiteness condition of being of type left-FP 1 exactly when $\omega^{\ell}$ is finitely generated.

Our investigations enable us to classify those semigroups such that $\omega^{\ell}$ is finitely generated that lie in certain important classes, such as strong semilattices of semigroups, inverse semigroups, Rees matrix semigroups (over semigroups) and completely regular semigroups. We consider closure properties for the class of semigroups such that $\omega^{\ell}$ is finitely generated, including under morphic image, direct product, semi-direct product, free product and 0-direct union. Our work was inspired by the stronger condition, stated for monoids in the work of White, of being pseudo-finite. Where appropriate, we specialise our investigations to pseudo-finite semigroups and monoids. In particular, we answer a question of Dales and White concerning the nature of pseudo-finite monoids.
\end{abstract}

Keywords Monoids - Semigroups $\cdot$ Left congruences $\cdot$ Finitely generated $\cdot \mathrm{FP}_{1}$. Pseudo-finite

Communicated by J. S. Wilson.

The research of Yang Dandan was supported by Grant No. 11501430 of the National Natural Science Foundation of China, and by Grant No. 20170604 of the Young Talents Project of Shaanxi Association for Science and Technology, and by Grant No. 20103176174 of the Fundamental Research Funds for the Central Universities. The research of Thomas Quinn-Gregson has been funded by a Postdoctoral Fellowship from the Department of Mathematics of the University of York and by the Deutsche Forshungsgemeinschaft. The research of Rida-e-Zenab has been funded by a Faculty for the Future Postdoctoral Fellowship from the Schlumberger Foundation.

Extended author information available on the last page of the article 
Mathematics Subject Classification 20 M 05 20 M 10

\section{Introduction}

A finitary condition for a class of (universal) algebras is a condition, defined in the appropriate language, that is satisfied by at least all finite members of the class. The concept was introduced and developed in the early part of the last century by Noether and Artin in their seminal work. Subsequently, finitary conditions have been of enormous importance in understanding the structure and behaviour of rings, groups, semigroups and many other kinds of algebras.

The classes of algebras we examine here are those of semigroups and monoids. The two finitary conditions we focus on may be stated in many different ways and arise from a variety of sources, as we explain in Sects. 2 and 3. The simplest way of approaching them is via the universal relation on a semigroup $S$, regarded as a left congruence. We remark that left ideals of semigroups are associated with left congruences, but, unlike the case for rings, not every left congruence comes from a left ideal. Left congruences on a monoid determine all monogenic representations by left actions on sets, in the standard way. We denote the universal left congruence relation on a semigroup $S$ by $\omega_{S}^{\ell}$; on occasion, where $S$ is not named, we write more simply $\omega^{\ell}$. The finitary conditions for $S$ that are the subject of this article are those of $\omega_{S}^{\ell}$ being finitely generated (as a left congruence) and the stronger condition of $S$ being pseudo-finite. Intuitively, the latter condition puts a bound on the length of the derivation required to relate two words, using only a finite set of relations; we give a precise definition in Sect. 2. It is well known and easy to see that if $G$ is a group, then $\omega_{G}^{\ell}$ is finitely generated if and only if $G$ is a finitely generated group. The work of [24] shows that $G$ is pseudo-finite if and only if $G$ is finite. For arbitrary monoids and semigroups, the situation is much more complex.

A semigroup $S$ is left noetherian if every left congruence is finitely generated; certainly then $\omega_{S}^{\ell}$ is finitely generated. The study of left noetherian semigroups was introduced by Hotzel in [11] and is still a developing topic [19]. At least in the monoid case, such a condition has been much exploited in the theory of acts over monoids. For example, if a monoid $M$ is left noetherian, then every finitely generated left $M$-act is finitely presented [20]. As shown in [17], if $S$ is left noetherian, then so is every subgroup of $S$. Our condition that $\omega_{S}^{\ell}$ is finitely generated may clearly be seen to be weaker than being left noetherian: it is easy to see that $\omega_{M}^{\ell}$ is finitely generated for any monoid $M$ with zero. More significantly, our characterisations of semigroups $S$ with $\omega_{S}^{\ell}$ being finitely generated have conditions that only refer to some 'top part' (merely the identity in the case of a monoid) and properties of a minimum ideal $I$, including conditions on the subgroups of $I$.

We remark that many other finitary conditions have been important in the study of semigroups and monoids, naturally including the properties of being finitely generated or finitely presented [23], and other finitary conditions on the lattices of one sided congruences, for example $[7,8]$, both of which arise from model-theoretic considerations. 
In the case of a monoid $M$ the condition that $\omega_{M}^{\ell}$ is finitely generated is equivalent to a number of notions that have arisen from a variety of sources. From a homological standpoint the concept of being of type left- $\mathrm{FP}_{n}$ was introduced for groups by Bieri [2] and later extended to monoids [4]. Much of the work into the general property of being of type left-FP ${ }_{n}$ has been in the case of groups, although a recent shift to monoids can be seen in $[9,10,15,16,21]$. Any monoid which possesses a finite complete rewriting system was shown by Anick [1] to be of type left-FP ${ }_{n}$ for each $n$. We refer the reader to [5] for a wider study. Using work of Kobayashi [15], we show that a monoid $M$ is of type left-FP ${ }_{1}$ exactly when $\omega_{M}^{\ell}$ is finitely generated. Moreover, $\omega_{M}^{\ell}$ being finitely generated may be stated in terms of the notion of ancestry introduced by White [24]. We present further formulations in Sect. 3.

The notion of being pseudo-finite was introduced in [24] in the language of ancestry. Theorem 1.7 of [24] shows that for a monoid $M$ the augmentation ideal $\ell_{1}^{0}(M)$ is finitely-generated if and only if $M$ is pseudo-finite. The work in [24] was motivated by the Dales-Żelazko conjecture, which states that a unital Banach algebra in which every maximal left ideal is finitely generated is necessarily finite dimensional. Through constructing links between the conjecture and ancestry, White showed the conjecture to be true for Banach algebras of the form $\ell^{1}(M)$ where $M$ is a weakly right cancellative monoid. In fact, it was a question posed to the second author by Dales and White [6], concerning the nature of pseudo-finite monoids, that led to this article. We answer the question in the negative in Example 7.7.

The objective of this paper is to make a comprehensive study of those semigroups $S$ such that $\omega_{S}^{\ell}$ is finitely generated, or $S$ is pseudo-finite. Our results often divide into two kinds: those for semigroups and those for monoids. On the way we consider numerous constructions, some of which have been considered before in the monoid case—see [10] for direct products, [18,22] for retracts and [9] for Clifford monoids. An important word of warning: the property of being of type left-FP 1 does not apply to semigroups. In [9] a semigroup is said to be of type left-FP 1 if $S^{1}$, the monoid obtained from $S$ by adjoining an identity if necessary, is of type left-FP 1 . However, for semigroups, the property of $\omega_{S}^{\ell}$ being finitely generated and that of $\omega_{S^{1}}^{\ell}$ being finitely generated differ considerably; see, for example, Corollaries 6.4 and 6.6.

The paper is organised as follows. In Sect. 2 we list some basic properties for a semigroup $S$ such that $\omega_{S}^{\ell}$ is finitely generated, and formally introduce the stronger property of being pseudo-finite. In Sect. 3 we explain the relationships between the conditions that $\omega_{S}^{\ell}$ is finitely generated, or $S$ is pseudo-finite, and a number of other notions such as ancestry, connected right Cayley graphs, type left- $\mathrm{FP}_{1}$ and right unitary generation by a subset. In Sect. 4 we consider the closure properties of the class of semigroups $S$ with $\omega_{S}^{\ell}$ being finitely generated, or of $S$ being pseudo-finite, under standard algebraic constructions: morphisms, direct products, semidirect products, free products and 0-direct unions. In Sect. 5 we focus on inverse semigroups, and establish several equivalent conditions for an inverse semigroup $S$ for $\omega_{S}^{\ell}$ to be finitely generated, or for $S$ to be pseudo-finite. We end the section by considering our properties for Brandt semigroups. Section 6 extends the results for Brandt semigroups to the much wider context of arbitrary Rees matrix semigroups over semigroups, with and without a zero and with or without an adjoined identity; the difference in the cases is striking. In Sect. 7 we return to considering constructions, this time two that are typical to 
semigroups: those of strong semilattices of semigroups and of Bruck-Reilly extensions. We also answer the question of Dales and White by constructing a particular semilattice of groups. Finally, in Sect. 8, we take a more global view. We consider semigroups possessing a completely simple minimum ideal (without further restriction), and then specialise to the class of completely regular semigroups (semigroups which are unions of groups) and then to bands (idempotent semigroups).

We have attempted to make the paper relatively self contained. We give a brief introduction to Green's relations in Sect.2. Many of the algebraic constructions we use will be standard, such as those of direct product or morphism. The details of those particular semigroups may be found in [13] or in the case of free products, in [3]. Our canonical notation for a semigroup is $S$ and for a monoid is $M$; the identity of a monoid $M$ is denoted by 1 . We write $\bar{X}$ to denote a set $\{(1, x): x \in X\}$ for a subset $X$ of a monoid $M$. For any $X \subseteq S$ we denote by $X^{2}$ both the set of pairs $X \times X$ and the set $\{x y: x, y \in S\}$, depending on the context, which we will always endeavour to make clear. The identity relation on any set $X$ is denoted by $\iota$ or $\iota_{X}$. Given a subset $A$ of $S^{2}$ for a semigroup $S$, the left congruence on $S$ generated by $A$ will be denoted by either $\rho_{A}$ or $\langle A\rangle$. The set of idempotents of a semigroup $S$ is denoted by $E(S)$.

\section{Preliminaries}

We make some initial observations surrounding the condition that $\omega^{\ell}$ is finitely generated. This leads naturally to the point where we can define the property of being pseudo-finite. We start with the following well-known result.

Lemma 2.1 [14, Lemma I.4.37] Let $S$ be a semigroup and $A$ be a subset of $S^{2}$. Then, for any $a, b \in S, a \rho_{A} b$ if and only if either $a=b$ or there exists a sequence

$$
a=t_{1} c_{1}, t_{1} d_{1}=t_{2} c_{2}, \ldots, t_{n} d_{n}=b
$$

where $t_{i} \in S^{1}$ and $\left(c_{i}, d_{i}\right) \in A \cup A^{-1}$ for all $1 \leq i \leq n$.

The sequence in the above lemma is referred to as an A-sequence of length $n$; if $n=0$, we interpret this sequence as being $a=b$.

Given a pair of left congruences $\delta$ and $\delta^{\prime}$ on $S$, we say that $\delta$ is a principal extension of $\delta^{\prime}$ if $\delta \supset \delta^{\prime}$ and there exists $(a, b) \in S^{2}$ such that $\delta=\left\langle\delta^{\prime} \cup\{(a, b)\}\right\rangle$. Clearly, if $\delta$ covers $\delta^{\prime}$ in the lattice of left congruences on $S$ then $\delta$ is a principal extension of $\delta^{\prime}$, but the converse is not true. This may be easily seen by observing that for any monoid $M$ with 0 , we have that $\omega_{M}^{\ell}=\langle\iota \cup\{1,0\}\rangle$, but $M$ may have non-trivial proper left congruences.

The proof of the next lemma is straightforward.

Lemma 2.2 Let $S$ be a semigroup. Then the following are equivalent:

(1) $\omega_{S}^{\ell}$ is finitely generated;

(2) there is a finite chain $\iota=\delta_{0} \subset \delta_{1} \subset \cdots \subset \delta_{n}=\omega_{S}^{\ell}$ of left congruences on $S$ where each $\delta_{i}$ is a principal extension of $\delta_{i-1}$ for all $1 \leq i \leq n$; 
(3) there exists a finite subset $X$ of $S$ such that $\omega_{S}^{\ell}=\left\langle X^{2}\right\rangle$;

(4) there exists a finite subset $X$ of $S$ such that for any $x \in X, \omega_{S}^{\ell}=\langle\{x\} \times X\rangle$;

(5) for any $u \in S$ there exists a finite subset $X$ of $S$ such that $u \in X$ and $\omega_{S}^{\ell}=$ $\langle\{u\} \times X\rangle$.

It follows from Lemma 2.2 that, for a semigroup $S$ with $\omega_{S}^{\ell}$ being finitely generated, we always have a generating set for $\omega_{S}^{\ell}$ of the form $X^{2}$ for some finite subset $X \subseteq S$. Moreover, in the case when $S$ is a monoid, we always have a generating set of the form $\bar{X}=\{(1, x): x \in X\}$ for some finite $X \subseteq S \backslash\{1\}$. We shall make use of this observation throughout the paper without reference.

Definition 2.3 Let $S$ be a semigroup with $\omega_{S}^{\ell}$ being generated by $A$, where $A \subseteq S^{2}$ is finite. We say that $S$ is pseudo-finite with respect to $A$ if there exists $n \in \mathbb{N}$ such that for any $a, b \in S$, there is an $A$-sequence from $a$ to $b$ of length at most $n$.

We say that $S$ is pseudo-finite with respect to $X$ if $X \subseteq S$ is finite and $S$ is pseudofinite with respect to $X^{2}$.

Remark 2.4 Let $S$ be a semigroup with $A \subseteq S^{2}$ finite. Then $S$ is pseudo-finite with respect to $A$ if and only if $\omega_{S}^{\ell}$ is the union of a finite chain of reflexive, symmetric relations $\rho_{A}^{n}$ where $a \rho_{A}^{n} b$ if there is an $A$-sequence of length at most $n$ relating $a$ to $b$.

The following lemma shows that the property of $\omega_{S}^{\ell}$ being generated by a finite set, or of $S$ being pseudo-finite with respect to a finite generating set, is independent of the given set of generators.

Lemma 2.5 Let $S$ be a semigroup and let $\omega_{S}^{\ell}$ be finitely generated by $H \subseteq S^{2}$. Suppose $\omega_{S}^{\ell}=\langle K\rangle$ for some $K \subseteq S^{2}$. Then there exists a finite subset $K^{\prime}$ of $K$ such that $\omega_{S}^{\ell}=\left\langle K^{\prime}\right\rangle$.

Further, if there exists $m \in \mathbb{N}$ such that for any $a, b \in S$, there is an $H$-sequence from a to $b$ of length at most $m$, then there is an $m^{\prime} \in \mathbb{N}$ such that for any $a, b \in S$, there is a $K^{\prime}$-sequence from a to $b$ of length at most $m^{\prime}$.

Proof The first statement is well known, but we give a short proof here for completeness and convenience.

We are given that $\omega_{S}^{\ell}=\langle H\rangle=\langle K\rangle$. Let $(h, k) \in H$. Then there is a $K$-sequence of length $n:=n(h, k)$

$$
h=t_{1} c_{1}, t_{1} d_{1}=t_{2} c_{2}, \ldots, t_{n} d_{n}=k,
$$

where $\left(c_{i}, d_{i}\right) \in K \cup K^{-1}$ and $t_{i} \in S^{1}$.

Let

$$
K_{(h, k)}=\left(\left\{\left(c_{1}, d_{1}\right), \ldots,\left(c_{n}, d_{n}\right)\right\} \cup\left\{\left(c_{1}, d_{1}\right), \ldots,\left(c_{n}, d_{n}\right)\right\}^{-1}\right) \cap K,
$$

so that $K_{(h, k)} \subseteq K,\left|K_{(h, k)}\right|<\infty$ and $(h, k) \in\left\langle K_{(h, k)}\right\rangle$. Let

$$
K^{\prime}=\bigcup_{(h, k) \in H} K_{(h, k)} .
$$


Since $H$ is finite, $K^{\prime}$ is a finite subset of $K$ and it is clear that $H \subseteq\left\langle K^{\prime}\right\rangle$. Thus

$$
\omega_{S}^{\ell}=\langle H\rangle \subseteq\left\langle K^{\prime}\right\rangle \subseteq \omega_{S}^{\ell}
$$

giving $\omega_{S}^{\ell}=\left\langle K^{\prime}\right\rangle$ as required.

Further, suppose there exists $m \in \mathbb{N}$ such that for any $a, b \in S$, there exists an $H$-sequence from $a$ to $b$ of length at most $m$, that is,

$$
a=s_{1} h_{1}, s_{1} k_{1}=s_{2} h_{2}, \ldots, s_{m} k_{m}=b,
$$

where $\left(h_{i}, k_{i}\right) \in H \cup H^{-1}$ and $s_{i} \in S^{1}$. Notice that for each $(h, k) \in H$, there is a $K^{\prime}$-sequence of length $n(h, k)$ for some $n \in \mathbb{N}$ connecting $h$ to $k$, and hence a sequence of the same length connecting $u h$ to $u k$, for any $u \in S^{1}$. Replace each $\left(s_{i} h_{i}, s_{i} k_{i}\right), 1 \leq i \leq m$ in above sequence with a $K^{\prime}$-sequence of length $n\left(h_{i}, k_{i}\right)$. Let

$$
m^{\prime}=m \times \max \{n(h, k):(h, k) \in H\} .
$$

Then there is a $K^{\prime}$-sequence from $a$ to $b$ of length at most $m^{\prime}$.

We make use of Lemma 2.5 in the definition below.

Definition 2.6 A semigroup $S$ is pseudo-finite if it is pseudo-finite with respect to some finite $A \subseteq S^{2}$, or equivalently, if it is pseudo-finite with respect to some finite $X \subseteq S$.

The following result is essentially folklore (see [24]):

Proposition 2.7 Let $G$ be a group and $A \subseteq G^{2}$. Then:

(1) $\omega_{G}^{\ell}$ is generated by $A$ if and only if $G$ is generated by $\left\{a^{-1} b:(a, b) \in A\right\}$;

(2) $G$ is pseudo-finite if and only if it is finite.

Let $M$ be monoid and let $B \subseteq M$. Then:

(3) if $M$ is generated by $B$, then $\omega_{M}^{\ell}$ is generated by $B \times\{1\}$.

Note that in Proposition 2.7, if $A$ is finite then certainly so is $\left\{a^{-1} b:(a, b) \in A\right\}$. On the other hand, if we have a finite set $C$ of generators of $G$, then $C \times\{1\}$ finitely generates $\omega_{G}^{\ell}$ using (3). We now give a way of extending Proposition 2.7(3) to semigroups.

Lemma 2.8 Let $S$ be a semigroup generated by $X \subseteq S$. Then $\omega_{S}^{\ell}$ is generated by $W_{X}^{2}$, where $W_{X}=X \cup\{x y: x, y \in X\}$. Moreover, if $X$ is finite, then so is $W_{X}$.

Proof Fix $x \in X$. For $x_{i_{1}} x_{i_{2}} \cdots x_{i_{k}} \in S$ where $x_{i_{j}} \in X, 1 \leq j \leq k$, we have

$$
\begin{aligned}
& x_{i_{1}} \cdots\left(x_{i_{k-1}} x_{i_{k}}\right) \rho_{W_{X}^{2}} x_{i_{1}} \cdots x_{i_{k-1}} \\
& \quad=x_{i_{1}} \cdots\left(x_{i_{k-2}} x_{i_{k-1}}\right) \rho_{W_{X}^{2}} \cdots \rho_{W_{X}^{2}} x_{i_{1}} x_{i_{2}} \rho_{W_{X}^{2}} x_{i_{1}} \rho_{W_{X}^{2}} x .
\end{aligned}
$$


In the above result, if $S=\bigcup_{1 \leq i \leq n} X^{i}$ for $n \in \mathbb{N}$, then clearly there is a bound on the length of $\rho_{W_{X}^{2}}$-sequences needed to connect elements of $S$, so that if $X$ is finite, then $S$ is finite and demonstrably, $S$ is pseudo-finite. The same comments apply to Proposition 2.7(3). However, we show in Example 5.5 that there exists a finitely generated monoid which is not pseudo-finite. On the other hand, if $M$ is any monoid with 0 , it is clear that $M$ is pseudo-finite with respect to $\{(1,0)\}$.

For what follows, it is convenient to recall a few details of Green's relations on a semigroup $S$, and their associated pre-orders; for further information, we recommend [13]. The relation $\leq_{\mathcal{L}}$ is defined on a semigroup $S$ by the rule

$$
a \leq_{\mathcal{L}} b \Leftrightarrow S^{1} a \subseteq S^{1} b
$$

It is easy to see that $\leq_{\mathcal{L}}$ is a right compatible pre-order, so that the associated equivalence relation $\mathcal{L}$ is a right congruence. The relations $\leq \mathcal{R}$ and $\mathcal{R}$ are defined dually, and the relations $\leq \mathcal{J}$ and $\mathcal{J}$ are obtained using principal two-sided ideals. The relation $\mathcal{H}$ is defined as $\mathcal{L} \cap \mathcal{R}$; any $\mathcal{H}$-class containing an idempotent $e$ is a maximal subgroup, denoted by $H_{e}$.

We now make some observations which will be very useful for later sections.

Lemma 2.9 Let $S$ be a non-trivial semigroup such that $\omega_{S}^{\ell}=\langle A\rangle$ for some $A \subseteq S^{2}$. Let $c(A)=\left\{x: \exists(x, y) \in A \cup A^{-1}\right\}$. Then for every $s \in S$ there exists some $x \in c(A)$ such that $s \leq \mathcal{L} x$.

Proof As $S$ is non-trivial we have $A \neq \varnothing$ and then $c(A) \neq \varnothing$. Let $s \in S$ and choose $u \in S$ with $s \neq u$. Since $s \rho_{A} u$, we have $s=t x$ for some $t \in S^{1}$ and $x \in c(A)$, giving $s \leq \mathcal{L} x$.

Proposition 2.10 Let $S$ be a semigroup. Then $\omega_{S}^{\ell}$ is finitely generated ( $S$ is pseudofinite) if and only if $\omega_{S^{1}}^{\ell}$ is finitely generated ( $S^{1}$ is pseudo-finite) and there is a finite set $U \subseteq S$ such that for every $a \in S$ we have $a \leq_{\mathcal{L}}$ u for some $u \in U$.

Proof It suffices to consider the case where $S$ is not a monoid.

Suppose first that $\omega_{S}^{\ell}$ is finitely generated by $A \subseteq S^{2}$. The subset $U$ exists by Lemma 2.9 and clearly $\omega_{S^{1}}^{\ell}$ is finitely generated by $A \cup\{(1, u)\}$ for any $u \in S$.

Conversely, suppose that $U$ exists as given and $\omega_{S^{1}}^{\ell}$ is finitely generated. Without loss of generality we can assume that $\bar{X}$ generates $\omega_{S^{1}}^{\ell}$ for some finite $X \subseteq S$. Let $Y=X^{2} \cup\{(u, u x): u \in U, x \in X\}$ so that $Y \subseteq S^{2}$ and is finite.

Let $s, t \in S$. We know that $s \rho_{\bar{X}} t$, so there exists an $\bar{X}$-sequence

$$
s=t_{1} c_{1}, t_{1} d_{1}=t_{2} c_{2}, \ldots, t_{n} d_{n}=t
$$

from $s$ to $t$. Let $z_{i}=t_{i} c_{i}$ for $1 \leq i \leq n$. Suppose first that no $z_{i}=1$ and consider $\left(t_{i} c_{i}, t_{i} d_{i}\right)$ for $1 \leq i \leq n$. Clearly $t_{i} \neq 1$ and so $t_{i}=p u$ for some $p \in S^{1}$ and $u \in U$. Then $\left(t_{i} c_{i}, t_{i} d_{i}\right)=\left(\right.$ puc $\left._{i}, p u d_{i}\right)$ and $\left(u c_{i}, u d_{i}\right) \in Y$. It follows that $s \rho_{Y} t$ in this case. The other situation is where $z_{i}=1$ for some $1<i \leq n$. Let $i$ be least such that $z_{i}=1$. We must have that $t_{i-1}=1=t_{i}=d_{i-1}=c_{i}$, so that $\left(c_{i-1}, d_{i-1}\right)=(x, 1)$ 
and $\left(c_{i}, d_{i}\right)=(1, y)$ for some $x, y \in X$. Then $s \rho_{Y} t_{i-1} c_{i-1}=x \rho_{Y} y=d_{i} t_{i}=z_{i+1}$, interpreting $z_{n+1}$ by $t$. An inductive argument now completes the proof that $s \rho_{Y} t$.

The claim involving pseudo-finiteness is clear from the argument above.

In dealing with semigroups such that $\omega_{S}^{\ell}$ is finitely generated we may, where convenient, call up the above description involving the monoid $S^{1}$.

The following result will be particularly useful when considering semigroups with a minimum ideal.

Lemma 2.11 Let $S$ be a semigroup and let I be a left ideal of $S$. Then $\omega_{S}^{\ell}$ is finitely generated if and only if there exists a finite subset $X$ of $S$ such that for every a $\in S$ we have some $x \in X$ with $a \leq_{\mathcal{L}} x$ and $\omega_{I}^{\ell}=\left.\rho_{X^{2}}\right|_{I \times I}$. In addition, $S$ is pseudo-finite if and only if there exists $n \in \mathbb{N}$ such that for any $a, b \in I$, there is an $X^{2}$-sequence from a to $b$ of length at most $n$.

Proof Suppose that $\omega_{S}^{\ell}=\left\langle X^{2}\right\rangle$ for some finite subset $X$ of $S$. Clearly, $\omega_{I}^{\ell}=\left.\rho_{X^{2}}\right|_{I \times I}$ and it follows from Lemma 2.9 that for every $a \in S$ there exists some $x \in X$ such that $a \leq_{\mathcal{L}} x$. Conversely, let $X$ be a finite subset of $S$ satisfying the required properties. Fix some $u \in I$ and let $Y=X \cup\{u\}$. For each $a \in S$, there exists $x \in X$ such that $a \leq_{\mathcal{L}} x$ and so $a=t x$ for some $t \in S^{1}$. It follows from the assumption $\omega_{I}^{\ell}=\left.\rho_{X^{2}}\right|_{I \times I}$ that $a=t x \rho_{Y^{2}} t u \rho_{Y^{2}} u$ as $u, t u \in I$, so that $\omega_{S}^{\ell}=\rho_{Y^{2}}$.

The second statement now follows from Lemma 2.5.

Corollary 2.12 Let $S$ be a monoid and let I be a left ideal of $S$. Then $\omega_{S}^{\ell}$ is finitely generated if and only if there exists a finite subset $X$ of $S$ such that $\omega_{I}^{\ell}=\left.\rho_{X^{2}}\right|_{I \times I}$. In addition, $S$ is pseudo-finite if and only if there exists $n \in \mathbb{N}$ such that for any $a, b \in I$, there is an $X^{2}$-sequence from a to $b$ of length at most $n$.

As a consequence of Lemma 2.11 we obtain the following extension of an existing result for monoids [9, Proposition 6]. This result also follows from the corresponding result for monoids in [9], together with Proposition 2.10. In the monoid case clearly we may drop the condition on the relation $\leq_{\mathcal{L}}$ below. Note also that the converse does not hold in general, as seen in [9, Example 1].

Corollary 2.13 Let $S$ be a semigroup and I be a left ideal of S. Suppose that $\omega_{I}^{\ell}$ is finitely generated ( $I$ is pseudo-finite) and there exists a finite subset X of $S$ such that for every $a \in S$ we have some $x \in X$ with $a \leq_{\mathcal{L}} x$. Then $\omega_{S}^{\ell}$ is finitely generated ( $S$ is pseudo-finite).

Corollary 2.14 The following are equivalent for a semigroup $S$ with zero:

(1) there exists a finite subset $X$ of $S$ such that for every a $\in S$ we have some $x \in X$ with $a \leq \mathcal{L} x$;

(2) $\omega_{S}^{\ell}$ is finitely generated;

(3) $S$ is pseudo-finite.

Proof Clearly, $\{0\}$ forms an ideal of $S$, and hence the required result holds by Lemma 2.11.

Note that in the above, $\omega_{S}^{\ell}$ is generated by $X \times\{0\}$. In the monoid case, this reduces to $\{(1,0)\}$, so, as we have already observed:

Corollary 2.15 Any monoid with zero is pseudo-finite. 


\section{Alternative conditions for $\omega^{\ell}$ to be finitely generated}

In this section we give a variety of alternative conditions for semigroups and monoids such that $\omega^{\ell}$ is finitely generated. For monoids these conditions are already known, involving the notions of ancestry [24], right Cayley graphs, the homological condition called type left-FP ${ }_{1}$, and of being right unitarily generated [15]. We remind the reader that in [9] a semigroup $S$ is said to be of type left-FP 1 if and only if the monoid $S^{1}$ is of type left-FP ${ }_{1}$. Although we do not attempt to consider here the property of being type left-FP ${ }_{1}$ for semigroups, we take an essentially different approach to [9]. Namely, for a semigroup $S$, we consider the property that $\omega_{S}^{\ell}$ is finitely generated, which is a stronger property than $\omega_{S^{1}}^{\ell}$ being finitely generated. Indeed, according to Corollary $2.15, \omega_{S^{1}}^{\ell}$ is finitely generated for any semigroup with 0 . On the other hand if $N$ is an infinite null semigroup, then as it has infinitely many maximal left ideals, Corollary 2.14 tells us that $\omega_{N}^{\ell}$ is not finitely generated.

Our first condition involves the notion of a left $M$-act over a monoid $M .{ }^{1}$ Let $S$ be a semigroup and let $A$ be a non-empty set. Then $A$ is a left $S$-act if there is a map $S \times A \rightarrow A$ where $(s, a) \mapsto s \cdot a$, such that for all $s, t \in S$ and $a \in A$ we have $s \cdot(t \cdot a)=(s t) \cdot a$. If $S=M$ is a monoid then we also insist that $1 \cdot a=a$ for all $a \in A$. The notion of a left $M$-act $A$ being finitely presented is the standard one from universal algebra, that is, $A$ is isomorphic to a finitely generated free left $M$-act factored by a finitely generated left $M$-act congruence. The free left $M$-act on one generator is isomorphic to $M$ regarded as a left ideal of itself, and as such, a left $M$-act congruence is precisely a left congruence on $M$. For further details of monoid and semigroup acts we refer the reader to the monograph [14].

Proposition 3.1 Let $M$ be a monoid. Then $\omega_{M}^{\ell}$ is finitely generated if and only if the trivial left $M$-act $\Theta_{M}=\{z\}$ is finitely presented.

Proof If $\omega_{M}^{\ell}$ is finitely generated then $\Theta_{M} \cong M / \omega_{M}^{\ell}$ is finitely presented.

Conversely, if $\Theta_{M}$ is finitely presented, then by a standard result of algebra, it has finite presentation with respect to any set of generators. Thus $\omega_{M}^{\ell}$, which is the kernel of the $M$-act morphism $M \rightarrow \Theta_{M}$, is finitely generated.

For the corresponding result for semigroups we need a little more care. Let $S$ be a semigroup. We say that an $S$-act A is quasi-free if $A$ is a disjoint union of copies of $S$, where $S$ is regarded as a left ideal of itself. To ease notation, we write $A=\bigcup_{i \in I} S_{i}$, where $S_{i}=\left\{s_{i}: s \in S\right\}, s_{i}=t_{j}$ if and only if $i=j$ and $s=t$, and for any $s_{i} \in A$ and $t \in S$ we have $t \cdot s_{i}=(t s)_{i}$.

Proposition 3.2 Let $S$ be a semigroup. Then $\omega_{S}^{\ell}$ is finitely generated if and only if the trivial $S$-act $\Theta_{S}$ is isomorphic to a quasi-free $S$-act $A=\bigcup_{i \in I} S_{i}$ where I is finite, factored by a finitely generated congruence.

Proof If $\omega_{S}^{\ell}$ is finitely generated then with $I=\{i\}$ and identifying $A=S_{1}$ with $S$ we have $S \rightarrow \Theta_{S}$ has kernel $\omega_{S}^{\ell}$.

\footnotetext{
$\overline{1 \text { The authors are grateful to Professor Nik Ruškuc for this observation. }}$
} 
Conversely, suppose that $\Theta_{S} \cong A / \rho$ where $A=\bigcup_{i \in I} S_{i}, \rho=\langle H\rangle$ and $I$ and $H$ are finite. Putting $K=\left\{(u, v): \exists\left(u_{i}, v_{j}\right) \in H\right.$ for some $\left.i, j \in I\right\}$, it is easy to see that $\rho_{K}=\omega_{S}^{\ell}$.

We next consider the notion of ancestry in a monoid due to White [24].

Definition 3.3 [24] Let $M$ be a monoid and let $X$ be a non-empty subset of $M$. We say that an element $a \in M$ has an ancestry of length $n$ with respect to $X$ if there exists a finite sequence $\left(z_{i}\right)_{i=1}^{n}$ of length $n$ in $M$ such that $z_{1}=a, z_{n}=1$ and for each $1<i \leq n$ there exists $x \in X$ such that either $z_{i} x=z_{i-1}$ or $z_{i}=z_{i-1} x$.

Lemma 3.4 Let $M$ be a monoid and let $X$ be a finite subset of $M$. Then $\omega_{M}^{\ell}$ is (finitely) generated by $\bar{X}$ ( $M$ is pseudo-finite with respect to $\bar{X})$ if and only if every element of $M$ has an ancestry (of bounded length) with respect to $X$.

Proof Suppose that $\omega_{M}^{\ell}=\rho_{\bar{X}}$. For any $a \in M$ we have $a \rho_{\bar{X}} 1$, so that there exists a sequence $a=t_{1} c_{1}, t_{1} d_{1}=t_{2} c_{2}, \ldots, t_{n} d_{n}=1$, where $t_{i} \in S^{1}$ and $\left(c_{i}, d_{i}\right) \in \bar{X} \cup \bar{X}^{-1}$ for all $1 \leq i \leq n$. Let $z_{1}=a, z_{n}=1$ and $z_{i}=t_{i} d_{i}$ for all $1<i<n$. If $\left(c_{i}, d_{i}\right)=(1, x) \in \bar{X}$ we have $z_{i-1}=t_{i} c_{i}=t_{i}$ and so $z_{i}=t_{i} d_{i}=z_{i-1} x$. On the other hand, if $\left(d_{i}, c_{i}\right)=(1, x) \in \bar{X}$ then $z_{i}=t_{i} d_{i}=t_{i}$ and so $z_{i-1}=t_{i} c_{i}=z_{i} x$. Hence $\left(z_{i}\right)_{i=1}^{n}$ is an ancestry of $a$ with respect to $X$ of length $n$.

Conversely, suppose that an element $a \in M$ has an ancestry of length $n$ with respect to $X$. Then there exists a finite sequence $\left(z_{i}\right)_{i=1}^{n}$ in $M$ such that $z_{1}=a, z_{n}=1$ and for $1<i \leq n$, there exists $x \in X$ such that $z_{i} x=z_{i-1}$ or $z_{i}=z_{i-1} x$. In the first case, $\left(z_{i-1}, z_{i}\right)=\left(z_{i} x, z_{i}\right)=\left(z_{i}, z_{i}\right)(x, 1)$ and in the second, $\left(z_{i-1}, z_{i}\right)=\left(z_{i-1}, z_{i-1} x\right)=$ $\left(z_{i-1}, z_{i-1}\right)(1, x)$. We thus obtain that $z_{1}=a, z_{2}, \ldots, z_{n}=1$ is a $\rho_{\bar{X}}$-sequence of length $n$ from $a$ to 1 . Hence $\omega_{M}^{\ell}=\rho_{\bar{X}}$.

The statement involving pseudo-finiteness is clear from the above.

We may restate the concept of ancestry by using right Cayley graphs.

Definition 3.5 Let $M$ be a monoid and $X$ a subset of $M$ (we do not assume that $X$ is a generating set for $M)$. The right Cayley graph $\Gamma^{r}(M, X)$ of $M$ with respect to $X$ is defined as follows:

(1) the vertex set is $M$;

(2) there is a directed edge labelled by $x \in X$ from $a$ to $b$, denoted by $a \stackrel{x}{\rightarrow} b$, if $b=a x$.

By definition, a right Cayley graph $\Gamma^{r}(M, X)$ is directed. However, underlying any right Cayley graph is an undirected labelled graph $\Gamma_{u}^{r}(M, X)$. We say that $\Gamma_{u}^{r}(M, X)$ is of bounded width if there is $n \in \mathbb{N}$ such that any two distinct vertices are joined by a path of length no greater than $n$; note that this implies $\Gamma_{u}^{r}(M, X)$ is connected.

Proposition 3.6 Let $M$ be a monoid and let $X$ be a finite subset of $M$. Then $\omega_{M}^{\ell}$ is finitely generated by $\bar{X}$ if and only if $\Gamma_{u}^{r}(M, X)$ is connected. Moreover, $M$ is pseudofinite with respect to $\bar{X}$ if and only if $\Gamma_{u}^{r}(M, X)$ has bounded width. 
Proof Suppose that $\omega_{M}^{\ell}=\rho_{\bar{X}}$. Let $a, b \in M$. Then there exists an $\bar{X}$-sequence

$$
a=t_{1} c_{1}, t_{1} d_{1}=t_{2} c_{2}, \ldots, t_{n} d_{n}=b
$$

where $\left(c_{i}, d_{i}\right) \in \bar{X} \cup \bar{X}^{-1}$ and $t_{i} \in M$ for all $1 \leq i \leq n$. Notice that for every pair $\left(t_{i} c_{i}, t_{i} d_{i}\right)$ we have a path in $\Gamma^{r}(M, X)$ of the form $t_{i} c_{i}=t_{i} \stackrel{d_{i}}{\rightarrow} t_{i} d_{i}$ if $c_{i}=1$, or $t_{i} c_{i} \stackrel{c_{i}}{\leftarrow} t_{i}=t_{i} d_{i}$ if $d_{i}=1$, so that $a$ is connected to $b$ in $\Gamma_{u}^{r}(M, X)$.

Conversely, suppose that $\Gamma_{u}^{r}(M, X)$ is connected. Suppose there exists an edge between a pair of elements $a$ and $b$ of $S$, so that we must have $a=b x$ or $b=a x$ for some $x \in X$ and $(a, b) \in \rho_{\bar{X}}$. Since any two vertices are connected by a path it follows that $\rho_{\bar{X}}=\omega_{M}^{\ell}$.

The second claim of the proposition is clear from the above proof.

Corollary 3.7 Let $S$ be a semigroup. Then $\omega_{S}^{\ell}$ is finitely generated (pseudo-finite) if and only if there exists some finite subset $X$ of $S$ such that $\Gamma_{u}^{r}\left(S^{1}, X\right)$ of $S^{1}$ is connected (has bounded width), and there is a finite set $U \subseteq S$ such that for every $a \in S$ we have $a \leq \mathcal{L} u$ for some $u \in U$.

Proof This follows from Propositions 2.10 and 3.6, together with the fact we may assume that if $\Gamma_{u}^{r}\left(S^{1}, X\right)$ is connected, then $1 \notin X$.

Another way to characterise a monoid $M$ with $\omega_{M}^{\ell}$ being finitely generated is to use a connection between right Cayley graphs and the property of being right unitarily generated. This equivalence was established by Kobayashi [15], together with the equivalence to the property of $M$ being type left-FP $\mathrm{F}_{1}$, as we now briefly explain. Further details may be found in [15].

Definition 3.8 [15] Let $M$ be a monoid and $N$ be a submonoid of $M$. Then $N$ is said to be right unitary if for any $n \in N$ and $m \in M$,

$$
m n \in N \Rightarrow m \in N
$$

Let $X$ be a subset of $M$ and let $U^{r}(X)$ denote the smallest right unitary submonoid of $M$ containing $X$. If $M=U^{r}(X)$, then $M$ is said to be right unitarily generated by $X$.

Definition 3.9 [15] Let $M$ be a monoid and $\mathbb{Z} M$ be the monoid ring of $M$ over the integers $\mathbb{Z}$. For $n \geqslant 0, M$ is of type left $-\mathrm{FP}_{n}$ if there is a resolution

$$
A_{n} \rightarrow A_{n-1} \rightarrow \cdots \rightarrow A_{1} \rightarrow A_{0} \rightarrow \mathbb{Z} \rightarrow 0
$$

of $\mathbb{Z}$, regarded as a left $\mathbb{Z} M$-module with trivial action, such that $A_{0}, A_{1}, \ldots, A_{n}$ are finitely generated left $\mathbb{Z} M$-modules.

Our next result, collecting together the equivalent conditions for $\omega_{M}^{\ell}$ to be finitely generated, follows from Proposition 3.2, Lemma 3.4 and Proposition 3.6, with the remaining conditions coming from [15, Proposition 2.4, Theorem 2.6]. 
Theorem 3.10 Let $M$ be a monoid with a finite subset $X$. The following conditions are equivalent:

(1) $\omega_{M}^{\ell}$ is finitely generated by $\bar{X}$;

(2) the trivial $M$-act $\Theta_{M}$ is isomorphic to $M / \rho_{\bar{X}}$ and so is finitely presented;

(3) each element of $M$ has an ancestry with respect to $X$;

(4) the undirected right Cayley graph $\Gamma_{u}^{r}(M, X)$ of $M$ with respect to $X$ is connected;

(5) $M$ is right unitarily generated by $X$.

Further, $M$ is of type left-FP 1 if and only if any (all) of these conditions hold.

Analogous omnibus results also hold for semigroups, and for pseudo-finite monoids and semigroups. In the case of being right unitarily generated, we would require a further concept of the number of steps involved in the generation of $M$.

\section{Standard constructions}

The aim of this section is to consider several standard constructions and their behaviour with respect to $\omega^{\ell}$ being finitely generated, and of being pseudo-finite. As usual, there are two kinds of questions one can ask: whether the class of semigroups with $\omega^{\ell}$ being finitely generated is closed under a particular construction, and whether the fact that $\omega^{\ell}$ is finitely generated passes down to components of the construction. The properties we look at include morphisms, direct products, semidirect products, free products and 0 -direct unions. We also provide a number of examples.

Our first result is known in the case of a retract of a monoid [21, Theorem 3].

Proposition 4.1 Let $S$ be a semigroup and let $T$ be a morphic image of $S$. If $\omega_{S}^{\ell}$ is finitely generated ( $S$ is pseudo-finite), then $\omega_{T}^{\ell}$ is finitely generated ( $T$ is pseudo-finite).

Proof Suppose that $\omega_{S}^{\ell}=\rho_{A}$ for some finite subset $A$ of $S^{2}$ and $\varphi: S \longrightarrow T$ is an epimorphism. For any $u, v \in T$, there exists $s, t \in S$ such that $s \varphi=u$ and $t \varphi=v$. Hence there exists a sequence $s=s_{1} a_{1}, s_{1} b_{1}=s_{2} a_{2}, \ldots, s_{n} b_{n}=t$ where $s_{i} \in S^{1}$ and $\left(a_{i}, b_{i}\right) \in A \cup A^{-1}$ for all $1 \leq i \leq n$. By applying $\varphi$ to the above sequence, we have

$$
u=s \varphi=\left(s_{1} \varphi\right)\left(a_{1} \varphi\right),\left(s_{1} \varphi\right)\left(b_{1} \varphi\right)=\left(s_{2} \varphi\right)\left(a_{2} \varphi\right), \ldots,\left(s_{n} \varphi\right)\left(b_{n} \varphi\right)=t \varphi=v
$$

giving that $\omega_{T}^{\ell}=\rho_{A \varphi}$ where $A \varphi=\{(u \varphi, v \varphi):(u, v) \in A\}$. Clearly, if $S$ is pseudofinite with respect to $A$, then $T$ is pseudo-finite with respect to $A \varphi$.

In [10], a direct product of a pair of monoids $M$ and $N$ is shown (in the context of being of type left-FP 1 ) to have the property that $\omega_{M \times N}^{\ell}$ is finitely generated if and only if both $\omega_{M}^{\ell}$ and $\omega_{N}^{\ell}$ are finitely generated. We now extend this result to cover pseudofiniteness. The first part of the lemma below follows immediately from Proposition 4.1, by applying the projection morphisms.

Proposition 4.2 Let $S$ and $T$ be semigroups. If $\omega_{S \times T}^{\ell}$ is finitely generated $(S \times T$ is pseudo-finite) then both $\omega_{S}^{\ell}$ and $\omega_{T}^{\ell}$ are finitely generated (pseudo-finite). If $S$ and $T$ are monoids, then the converse is true. 
Proof Let $S$ and $T$ be monoids such that $\omega_{S}^{\ell}=\rho_{X^{2}}$ and $\omega_{T}^{\ell}=\rho_{Y^{2}}$ for some finite subsets $X \subseteq S$ and $Y \subseteq T$. For any $(s, t),(u, v) \in S \times T$, we have $s=s_{1} a_{1}, s_{1} b_{1}=$ $s_{2} a_{2}, \ldots, s_{m} b_{m}=u$ where $m \in \mathbb{N}^{0}, s_{i} \in S^{1},\left(a_{i}, b_{i}\right) \in X^{2}$ for all $1 \leq i \leq m$, and $t=t_{1} c_{1}, t_{1} d_{1}=t_{2} c_{2}, \ldots, t_{n} d_{n}=v$ where $n \in \mathbb{N}^{0}, t_{i} \in T^{1},\left(c_{i}, d_{i}\right) \in \bar{Y}^{2}$ for all $1 \leq i \leq n$. If $n \geqslant m$, then we put $s_{m+1}=\cdots=s_{n}=s_{m}$ and $a_{m+1}=b_{m+1}=\cdots=$ $a_{n}=b_{n}=b_{m}$. Then

$$
(s, t)=\left(s_{1} a_{1}, t_{1} c_{1}\right),\left(s_{1} b_{1}, t_{1} d_{1}\right)=\left(s_{2} a_{2}, t_{2} c_{2}\right), \ldots,\left(s_{n} b_{n}, t_{n} d_{n}\right)=(u, v),
$$

so that

$$
(s, t)=\left(s_{1}, t_{1}\right)\left(a_{1}, c_{1}\right),\left(s_{1}, t_{1}\right)\left(b_{1}, d_{1}\right)=\left(s_{2}, t_{2}\right)\left(a_{2}, c_{2}\right), \ldots,\left(s_{n}, t_{n}\right)\left(b_{n}, d_{n}\right)=(u, v) .
$$

A similar discussion holds for $n<m$, so that $\omega_{S \times T}^{\ell}=\rho_{(X \times Y)^{2}}$. Since the length of the $(X \times Y)^{2}$-sequence required is no greater than the maximum of $m$ and $n$, it is clear that the statement on pseudo-finiteness also holds.

The converse of Proposition 4.2 does not necessarily hold if we remove the condition that $S$ and $T$ are monoids.

Example 4.3 Let $C$ be the infinite cyclic monoid generated by $a$. It follows from Proposition 2.7 we have $\omega_{C}^{\ell}=\rho_{A}$ where $A=\left\{\left(a, a^{2}\right)\right\}$. For any $i \in \mathbb{N}$, if $\left(a, a^{i}\right)=s\left(a^{j}, a^{k}\right)$ where $s \in C^{2}$ we have $s=(1,1)$ and $\left(a, a^{i}\right)=\left(a^{j}, a^{k}\right)$. Lemma 2.9 now says $\omega_{C \times C}^{\ell}$ is not finitely generated.

Let $S$ and $T$ be monoids such that $T$ acts on $S$. If $t \cdot\left(s s^{\prime}\right)=(t \cdot s)\left(t \cdot s^{\prime}\right)$ for all $t \in T$ and $s, s^{\prime} \in S$, then we say that $T$ acts on $S$ by morphisms. In this case we can form the semidirect product $S \rtimes T$ with underlying set $S \times T$ and binary operation given by $(s, t)\left(s^{\prime}, t^{\prime}\right)=\left(s\left(t \cdot s^{\prime}\right), t t^{\prime}\right)$. It is easy to see that $S \rtimes T$ is a semigroup, and if $T$ acts monoidally, that is, if $t \cdot 1_{S}=1_{S}$ for all $t \in T$, then $S \rtimes T$ is a monoid with identity $(1,1):=\left(1_{S}, 1_{T}\right)$.

Proposition 4.4 Let $S$ and $T$ be monoids such that $\omega_{S}^{\ell}=\left\langle U^{2}\right\rangle$ and $\omega_{T}^{\ell}=\left\langle V^{2}\right\rangle$ for some finite subsets $U \subseteq S$ and $V \subseteq T$. Suppose $T$ acts monoidally on $S$ by morphisms. Then $\omega_{S \rtimes T}^{\ell}$ is finitely generated. Moreover, if $S$ and $T$ are pseudo-finite, then so is $S \rtimes T$.

Proof Let $W=P \cup Q$ where $P=\left\{\left((s, 1),\left(s^{\prime}, 1\right)\right): s, s^{\prime} \in U\right\}$ and $Q=$ $\left\{\left((1, t),\left(1, t^{\prime}\right)\right): t, t^{\prime} \in V\right\}$. We claim that $\omega_{S \rtimes T}^{\ell}=\langle W\rangle$. For this we notice that if $u \in S$ is connected to $1 \in S$ via a $U^{2}$-sequence of length $n$, then $(u, 1)$ is connected to $(1,1)$ in $S \rtimes T$ via a $P$-sequence of the same length. A similar statement holds for $T$ and $Q$. Now let $(s, t) \in S \rtimes T$. Then

$$
(s, t)=(s, 1)(1, t) \rho_{W}(s, 1)(1,1)=(s, 1) \rho_{W}(1,1)
$$

and so the required results hold. 
Since $T$ is a morphic image of $S \rtimes T$, if $\omega_{S \rtimes T}^{\ell}$ is finitely generated ( $S \rtimes T$ is pseudo-finite), then $\omega_{T}^{\ell}$ is finitely generated ( $T$ is pseudo-finite). In the case of a direct product, we know that if $\omega_{S \times T}^{\ell}$ is finitely generated then so is $\omega_{S}^{\ell}$. For arbitrary semidirect products, this need not hold, as we will see in Example 5.6.

The following example shows that in Proposition 4.4, the action of $T$ on $S$ being monoidal is necessary.

Example 4.5 Let $X$ be a finite set and let $X^{*}$ be the free monoid generated by $X$. By Proposition 2.7, $\omega_{X^{*}}^{\ell}$ is finitely generated. Let $\mathcal{Y}$ be the join semilattice consisting of all finite subsets of $X^{*}$ including the empty word $\epsilon$ under union and let $\mathcal{Y}^{0}=\mathcal{Y} \cup\{0\}$. Since $\mathcal{Y}^{0}$ has identity $\{\epsilon\}$ and a zero, $\omega_{\mathcal{Y}^{\ell}}^{\ell}$ is finitely generated by Corollary 2.15. Define an action of $X^{*}$ on $\mathcal{Y}^{0}$ as follows:

$$
w \cdot 0=0, w \cdot A=w A=\{w a: a \in A\} \text { for } w \in X^{*} \text { and } A \in \mathcal{Y} .
$$

It is easy to check that this is an action by morphisms, but is not a monoid action because $w \cdot\{\epsilon\}=\{w\} \neq\{\epsilon\}$. Put $T=\mathcal{Y}^{0} \rtimes X^{*}$. Let $x \in X$ and, for each $i \in \mathbb{N}$, suppose $\left(\{\epsilon\}, x^{i}\right)=(B, u)\left(Y, w_{i}\right)$ for some $(B, u) \in T^{1}$ and $\left(Y, w_{i}\right) \in T$. Then $\{\epsilon\}=B \cup(u \cdot Y)$, so that $B=u \cdot Y=\{\epsilon\}$, giving $u=\epsilon$, and $w_{i}=x^{i}$. Hence $T$ has infinitely many maximal $\mathcal{L}$-classes, and $\omega_{T}^{\ell}$ is not finitely generated by Lemma 2.9 .

For the next result it is convenient to have the notion of length $|w|$ of an element $w$ in the free product $S * T$ of semigroups $S$ and $T$. We say that $w$ has length $n$ if $w=a_{1} a_{2} \ldots a_{n}$ where, if $a_{i} \in S$ (respectively, $T$ ), then $a_{i+1} \in T$ (respectively, $S$ ). Notice that $\left|w w^{\prime}\right|=|w|+\left|w^{\prime}\right|$ or $|w|+\left|w^{\prime}\right|-1$.

Proposition 4.6 Let $S$ and $T$ be semigroups. Then $\omega_{S * T}^{\ell}$ is finitely generated if and only if $\omega_{S}^{\ell}$ and $\omega_{T}^{\ell}$ are finitely generated. The semigroup $S * T$ is never pseudo-finite.

Proof Suppose first that $\omega_{S}^{\ell}=\rho_{U^{2}}$ and $\omega_{T}^{\ell}=\rho_{V^{2}}$ where $U$ and $V$ are finite. Fix $u \in U$ and $v \in V$ and put $W=U \cup V$; note that $u \rho_{W^{2}} v$. We claim that $\omega_{S * T}^{\ell}=\rho_{W^{2}}$. If $w$ is an element of length 1 in $S * T$ then either $w \in S$, so that as $w \rho_{U^{2}} u$ in $S$, we also have $w \rho_{W^{2}} u$ in $S * T$; similarly if $w \in T$. Suppose now that $n>1$ and any element of length $n-1$ is $\rho_{W^{2}}$-related to $u$ (or, equivalently, to $v$ ). Let $w=a_{1} a_{2} \ldots a_{n} \in S * T$ have length $n$. By our inductive hypothesis, $w \rho_{W^{2}} a_{1} u$ and $w \rho_{W^{2}} a_{1} v$. Since one of $a_{1} u, a_{1} v$ has length 1 , we have completed our claim.

Conversely, suppose that $\omega_{S * T}^{\ell}$ is finitely generated by $P^{2}$. Consider the monoid $S^{1}$. Clearly $S$ embeds into $S^{1}$ and there is the trivial morphism $T \rightarrow S^{1}$ that takes every element of $T$ to 1 . By the nature of free products, these morphisms can be simultaneously extended to a morphism from $S * T$ to $S^{1}$ that is clearly onto. By Proposition $4.1, \omega_{S^{1}}^{\ell}$ is finitely generated. Either $S$ is trivial, so that clearly $\omega_{S}^{\ell}$ is finitely generated, or we can find distinct $s, u \in S$. In the latter case there exists a $P^{2}$ sequence connecting $s$ to $u$, of which the first step gives $s=t c$ for some $t \in(S * T)^{1}$ and $c \in P$. It follows that (with a natural identification) we have $t \in S^{1}$ and $c \in S$. From Proposition 2.10 we deduce $\omega_{S}^{\ell}$ is finitely generated, and similarly for $\omega_{T}^{\ell}$.

To see that $S * T$ is never pseudo-finite, suppose for contradiction that $S * T$ is pseudo-finite with respect to $P^{2}$, and the bound on the length of the $P^{2}$-sequences 
needed to connect elements of $S * T$ is $k$. Let $h^{t}, h^{b}$ be the lengths of the longest and shortest elements of $P$, respectively. Consider $t \in(S * T)^{1}$ and $c, d \in P$. Then

$$
|| t c|-| t d||=|| t|+| c|-| t|-| d| \pm 1|=|| c|-| d| \pm 1| \leq h^{t}-h^{b}+1 .
$$

Let $w \in S * T$ have length $r$ where $r>k\left(h^{t}-h^{b}+1\right)$. It is now clear that $w$ cannot be related to any element of length 1 by a $P^{2}$-sequence of length $k$. Thus $S * T$ cannot be pseudo-finite.

Turning our attention to the case of the monoid free product of two monoids $M *^{m} N$ (where, of course, the monoid identities are identified, so that $M *^{m} N=$ $M * N /\left\langle\left(1_{M}, 1_{N}\right)\right\rangle$, we note from [16, Proposition 4.1] that if $M$ and $N$ are finitely presented, then $\omega_{M *^{m} N}^{\ell}$ is finitely generated if $\omega_{M}^{\ell}$ and $\omega_{N}^{\ell}$ are. A similar argument to that in Proposition 4.6 allows us to extend this to arbitrary monoids. The new complication is that although length is still well defined, the length of a product of $w$ and $v$ may be less than $|w|+|v|-1$, since right cancellative elements at the end of $w$ may cancel with left cancellative elements at the start of $v$. Bearing this in mind, we can adapt the proof of Proposition 4.6 to show:

Corollary 4.7 Let $M$ and $N$ be monoids. Then $\omega_{M *^{m} N}^{\ell}$ is finitely generated if and only if $\omega_{M}^{\ell}$ and $\omega_{N}^{\ell}$ are finitely generated. The monoid $M *^{m} N$ is never pseudo-finite unless one of $M, N$ is pseudo-finite and the other is trivial.

Proof The first statement follows as in Proposition 4.6. For the second, suppose for contradiction that $M *^{m} N$ is pseudo-finite with respect to $P^{2}$, and the bound on the length of the $P^{2}$-sequences needed to connect elements of $M *^{m} N$ is $k$. Let $h$ be the length of the longest element of $P$. Notice if $a \in M *^{m} N$ and $b \in P$ then $|a b|=|a|+p$ where $-h \leq p \leq h$. Consider $t \in M *^{m} N$ and $c, d \in P$. Then there exists $-h \leq p, q \leq h$ such that $|t c|=|t|+p$ and $|t d|=|t|+q$, and so

$$
|| t c|-| t d||=|(|t|+p)-(|t|+q)|=|p-q| \leq 2 h .
$$

The proof then follows that of Proposition 4.6 by taking $w$ to have length $r>2 \mathrm{kh}$.

Finally, if neither monoid is trivial then an argument as in Proposition 4.6, adjusted as indicated above, gives that $M *^{m} N$ is not pseudo-finite. Without loss of generality suppose that $N$ is trivial. Then $M *^{m} N$ is isomorphic to $M$ and so is pseudo-finite if and only if $M$ is.

We end this section by considering 0 -direct unions. We only need consider semigroups, since any monoid with 0 is pseudo-finite.

Proposition 4.8 Let $S$ and $T$ be semigroups with zero and let $P=S \cup T$ be the 0 direct union of $S$ and $T$. Then $\omega_{P}^{\ell}$ is finitely generated if and only if both $\omega_{S}^{\ell}$ and $\omega_{T}^{\ell}$ are finitely generated. Moreover, $P$ is pseudo-finite if and only if both $S$ and $T$ are pseudo-finite.

Proof Recall from Corollary 2.14 that for a semigroup $S$ with zero, $\omega_{S}^{\ell}$ is finitely generated if and only if $S$ is pseudo-finite. 
If $S$ and $T$ are pseudo-finite, then there are finite subsets $U$ of $S$ ( $V$ of $T$ ) such that for every $a \in S(b \in T)$ there is some $u \in U(v \in V)$ such that $a \leq \mathcal{L} u(b \leq \mathcal{L} v)$. Let $W=U \cup V$; it is then clear that for every $p \in P$ we have $p \leq_{\mathcal{L}} w$ for some $w \in W$. Corollary 2.14 gives that $P$ is pseudo-finite.

For the converse, we need only remark that $S$ and $T$ are morphic images of $P$, and invoke Proposition 4.1.

By a simple induction argument we note that the result above holds for the 0-direct union of finitely many semigroups.

\section{Inverse semigroups}

Inverse monoids $M$ such that $\omega_{M}^{\ell}$ is finitely generated were briefly considered in [9] in the case where $M$ has a least idempotent. In the case where $M$ is a semilattice of groups, this latter condition follows from the fact that $\omega_{M}^{\ell}$ is finitely generated, however, as we show, it is not necessary for $M$ to have a least idempotent in order that $\omega_{M}^{\ell}$ is finitely generated. Our focus in this section is to give a complete characterisation of those inverse semigroups $S$ such that $(i) \omega_{S}^{\ell}$ is finitely generated, and ( $\left.i i\right) S$ is pseudo-finite.

We begin by remarking that if $S$ is an inverse semigroup and $\omega_{S}^{\ell}$ is generated by $A \subseteq S^{2}$, then the universal relation, regarded as a right congruence and denoted by $\omega_{S}^{r}$, is generated by $A_{1}=\left\{\left(a^{-1}, b^{-1}\right):(a, b) \in A\right\}$ and so $\omega_{S}^{\ell}$ is finitely generated if and only if $\omega_{S}^{r}$ is finitely generated.

Theorem 5.1 Let $S$ be an inverse semigroup and $E(S)$ be the set of idempotents of $S$. Then the following statements are equivalent:

(1) $\omega_{S}^{\ell}$ is finitely generated;

(2) (i) there is a finite set $U \subseteq E(S)$ such that for every $e \in E(S)$ we have $e \leq u$ for some $u \in U$; and

(ii) there is a finitely generated inverse subsemigroup $W$ of $S$ such that for all $a \in S$ and $e \in E(W)$, there exists $w \in W$ with $a w=e w^{-1} w$;

(3) (i) there is a finite set $U \subseteq E(S)$ such that for every $e \in E(S)$ we have $e \leq u$ for some $u \in U$; and

(iii) there is a finitely generated inverse subsemigroup $W$ of $S$ such that for all $a \in S$ there exists $w \in W$ with aw $\in E(W)$.

Proof $(1) \Rightarrow$ (2) Condition ( $i$ ) follows easily from Proposition 2.10 and the fact that $S$ is inverse.

To show ( $i i)$, suppose that $\omega_{S}^{\ell}=\left\langle X^{2}\right\rangle$ for some finite subset $X \subseteq S$. Let $W$ be the subsemigroup of $S$ generated by $X \cup X^{-1}$ where $X^{-1}$ is the set of all inverses of elements in $X$. First, we show that, for any $a, b \in S$, a finite sequence

$$
b=s_{1} p_{1}, s_{1} q_{1}=s_{2} p_{2}, \ldots, s_{n} q_{n}=a
$$


where $s_{i} \in S^{1}, p_{i}, q_{i} \in X$ for $1 \leq i \leq n$, gives as $=b s^{-1} s$ with $s=$ $q_{n}^{-1} p_{n} \cdots q_{1}^{-1} p_{1}$. If $n=1$, then

$$
a s=a q_{1}^{-1} p_{1}=s_{1} q_{1} q_{1}^{-1} p_{1}=s_{1} p_{1} p_{1}^{-1} q_{1} q_{1}^{-1} p_{1}=b p_{1}^{-1} q_{1} q_{1}^{-1} p_{1}=b s^{-1} s .
$$

Suppose that the result holds for $n=k$. We show the result also holds for $n=k+1$. Here we have

$$
b=s_{1} p_{1}, s_{1} q_{1}=s_{2} p_{2}, \ldots, s_{k} q_{k}=s_{k+1} p_{k+1}, s_{k+1} q_{k+1}=a .
$$

Put $s_{k} q_{k}=s_{k+1} p_{k+1}=c$. Then, by induction,

$$
c\left(q_{k}^{-1} p_{k} \cdots q_{1}^{-1} p_{1}\right)=b\left(q_{k}^{-1} p_{k} \cdots q_{1}^{-1} p_{1}\right)^{-1}\left(q_{k}^{-1} p_{k} \cdots q_{1}^{-1} p_{1}\right) .
$$

Now we have

$$
\begin{aligned}
a( & \left.q_{k+1}^{-1} p_{k+1} q_{k}^{-1} p_{k} \cdots q_{1}^{-1} p_{1}\right) \\
= & s_{k+1} q_{k+1}\left(q_{k+1}^{-1} p_{k+1} q_{k}^{-1} p_{k} \cdots q_{1}^{-1} p_{1}\right) \\
= & s_{k+1} p_{k+1}\left(p_{k+1}^{-1} q_{k+1} q_{k+1}^{-1} p_{k+1}\right)\left(q_{k}^{-1} p_{k} \cdots q_{1}^{-1} p_{1}\right) \\
= & s_{k+1} p_{k+1}\left(q_{k}^{-1} p_{k} \cdots q_{1}^{-1} p_{1}\right)\left(q_{k}^{-1} p_{k} \cdots q_{1}^{-1} p_{1}\right)^{-1} \\
& \left(p_{k+1}^{-1} q_{k+1} q_{k+1}^{-1} p_{k+1}\right)\left(q_{k}^{-1} p_{k} \cdots q_{1}^{-1} p_{1}\right) \\
= & c\left(q_{k}^{-1} p_{k} \cdots q_{1}^{-1} p_{1}\right)\left(q_{k}^{-1} p_{k} \cdots q_{1}^{-1} p_{1}\right)^{-1} \\
& \left(p_{k+1}^{-1} q_{k+1} q_{k+1}^{-1} p_{k+1}\right)\left(q_{k}^{-1} p_{k} \cdots q_{1}^{-1} p_{1}\right) \\
= & b\left(q_{k}^{-1} p_{k} \cdots q_{1}^{-1} p_{1}\right)^{-1}\left(q_{k}^{-1} p_{k} \cdots q_{1}^{-1} p_{1}\right) \\
& \left(q_{k}^{-1} p_{k} \cdots q_{1}^{-1} p_{1}\right)^{-1}\left(p_{k+1}^{-1} q_{k+1} q_{k+1}^{-1} p_{k+1}\right)\left(q_{k}^{-1} p_{k} \cdots q_{1}^{-1} p_{1}\right) \\
= & b\left(q_{k}^{-1} p_{k} \cdots q_{1}^{-1} p_{1}\right)^{-1}\left(p_{k+1}^{-1} q_{k+1} q_{k+1}^{-1} p_{k+1}\right)\left(q_{k}^{-1} p_{k} \cdots q_{1}^{-1} p_{1}\right) \\
= & b\left(q_{k+1}^{-1} p_{k+1} q_{k}^{-1} p_{k} \cdots q_{1}^{-1} p_{1}\right)^{-1}\left(q_{k+1}^{-1} p_{k+1} q_{k}^{-1} p_{k} \cdots q_{1}^{-1} p_{1}\right) .
\end{aligned}
$$

Choosing $b=e \in E(W)$ we have $a s=e s^{-1} s$.

(2) $\Rightarrow$ (3) is clear.

(3) $\Rightarrow$ (1) Suppose that $W$ is a finitely generated inverse subsemigroup of $S$ with a finite set $Y=Y^{-1}$ of generators, and $U$ is the set of all idempotents guaranteed by $(i)$. We show that $\omega_{S}^{\ell}=\left\langle H^{2}\right\rangle$ where $H=Y \cup U$. Let $w_{1} w_{2} \cdots w_{k}$ be a finite product of elements in $Y$. Then for each $1 \leq i \leq k$ there exists some $e_{i} \in U$ such that $w_{i}=w_{i} e_{i}$, and so

$$
\begin{aligned}
& e_{1} \rho_{H^{2}} w_{1}=w_{1} e_{1} \rho_{H^{2}} w_{1} w_{2} \rho_{H^{2}} \cdots \rho_{H^{2}} w_{1} w_{2} \cdots w_{k-1} \\
& =w_{1} w_{2} \cdots w_{k-1} e_{k-1} \rho_{H^{2}} w_{1} w_{2} \cdots w_{k-1} w_{k} .
\end{aligned}
$$

For any $a \in S$, by assumption, there exists $w \in W$ such that $a w \in W$. By taking $e \in U$ such that $a \leq_{\mathcal{L}} e$, we have $a=a e \rho_{H^{2}} a w \rho_{H^{2}} e$, so $\rho_{H^{2}}=\omega_{S}^{\ell}$, as required. 
Corollary 5.2 Let $S$ be an inverse monoid $S$. Then $\omega_{S}^{\ell}$ is finitely generated if and only if (ii) or (iii) of Theorem 5.1 hold.

Now we specialise Theorem 5.1 to the pseudo-finite case. We first make the following observation: if $S$ is an inverse semigroup and the semilattice $E(S)$ of idempotents of $S$ has least element (zero) $e$, then $H_{e}=e S e$. Indeed, for any $s \in S$,

$$
\text { ese } \mathcal{R} e\left(\operatorname{ses}^{-1}\right)=e \text { and ese } \mathcal{L}\left(s^{-1} e s\right) e=e
$$

so that ese $\mathcal{H} e$ and so $e S e \subseteq H_{e}$. Clearly, $H_{e} \subseteq e S e$ and so $H_{e}=e S e$.

Proposition 5.3 Suppose that $S$ is an inverse semigroup with semilattice of idempotents $E(S)$. Then $S$ is pseudo-finite if and only if there is a finite set $U \subseteq E(S)$ such that for every $f \in E(S)$ we have $f \leq u$ for some $u \in U ; E(S)$ has a least element e, and the group $\mathcal{H}$-class $H_{e}$ is finite.

Proof Suppose that $S$ is pseudo-finite with respect to $X \subseteq S$. The first condition follows from Theorem 5.1. For any pair of idempotents $f, g \in E(S)$, there exists an $X^{2}$-sequence

$$
g=t_{1} c_{1}, t_{1} d_{1}=t_{2} c_{2}, \ldots, t_{n} d_{n}=f
$$

where $t_{i} \in S,\left(c_{i}, d_{i}\right) \in X^{2}$ for $1 \leq i \leq n$. It follows from the proof of Theorem 5.1 that $f h=g h^{-1} h$ where $h=d_{n}^{-1} c_{n} \cdots d_{1}^{-1} c_{1}$. As $S$ is an inverse semigroup, $f h=g h^{-1} h$ gives $f h(f h)^{-1}=g h^{-1} h\left(g h^{-1} h\right)^{-1}$, and so $f h h^{-1}=g h^{-1} h$. Note that every idempotent $f, g \in E(S)$ leads to such an idempotent $h^{-1} h$. As $S$ is pseudo-finite, we can bound the length of the $X^{2}$-sequences required and find a finite set $H$ consisting of those $h$ obtained as above. Let $w$ be the product of all $h^{-1} h$ such that $h \in H$. Then $f w=g w$, and so $f g w=g w$, giving $e=g w$ is least element for $E(S)$ and $H_{e}=e S e$. Further, $H_{e}$ is a morphic image of $S$ via $\varphi: S \rightarrow H_{e}$ defined by $s \varphi=e s e$. Indeed, for any $a, b \in S$,

$$
a e b e=a e b b^{-1} b e=a b\left(b^{-1} e b\right) e=a b e,
$$

and so

$$
(a \varphi)(b \varphi)=(e a e)(e b e)=e a e b e=e a b e=(a b) \varphi
$$

By Proposition 4.1, $H_{e}$ is pseudo-finite and hence a finite group by Proposition 2.7.

Conversely, suppose that $E(S)$ has a least element $e, H_{e}=e S e$ is finite and $U$ exists as given; put $Y=H_{e} \cup U$. For any $a \in S$,

$$
e=\left(a^{-1} e a\right) e=a^{-1}\left(e a a^{-1} a e\right)=a^{-1}\left(e a e a^{-1} a\right)=a^{-1} e\left(a e a^{-1}\right) a=a^{-1} e a
$$

giving $a e=a a^{-1} e a=e a$ and so $e a e=a e$. Moreover, there exists $f \in U$ such that $a \leq \mathcal{L} f$, so that $a=$ af $\rho_{Y^{2}}$ ae $=$ eae $\rho_{Y^{2}} e$. Clearly then $S$ is pseudo-finite with respect to $Y$. 
Clearly in the case of a monoid we may drop the first condition on Proposition 5.3. We also have the following consequence of Theorem 5.1.

Corollary 5.4 [9, Corollary 4]. Let $M$ be an inverse monoid with a least idempotent e. Then $\omega_{M}^{\ell}$ is finitely generated if and only if $H_{e}$ is finitely generated.

Our next example shows that the existence of a least idempotent is not necessary for $\omega^{\ell}$ to be finitely generated.

Example 5.5 Consider the Bicyclic monoid $B$. Then $\omega_{B}^{\ell}$ is finitely generated but $B$ is not pseudo-finite.

Proof Regarding the underlying set of $B$ as $\mathbb{N}^{0} \times \mathbb{N}^{0}$, it is clear that $B$ is finitely generated by $\{(0,1),(1,0)\}$ and so $\omega_{B}^{\ell}$ is finitely generated by Proposition 2.7 . The idempotents of $B$ form an infinite descending chain, and thus $B$ is not pseudo-finite by Corollary 5.4 .

For any monoid $M$ with a finitely generated minimum ideal, we have by [9, Proposition 6] and Proposition 2.7 that $\omega_{M}^{\ell}$ is finitely generated. We have seen that a pseudo-finite inverse semigroup possesses a minimum ideal which is finitely generated (in fact finite). The following example shows that the same is not true for inverse semigroups $S$ satisfying the weaker condition that $\omega_{S}^{\ell}$ is finitely generated.

Example 5.6 Let $G$ be the infinite cyclic group on $g$ and let $Y=\left\{e_{i}: i \in \mathbb{Q}\right\} \cup\{1\}$ be a semilattice, with $e_{i} e_{j}=e_{k}$ where $k=\min \{i, j\}$ and 1 is the identity. Define an action of $G$ on $Y$ by

$$
g^{i} \cdot 1=1, g^{i} \cdot e_{j}=e_{i+j}
$$

It is easy to see that this is an action by morphisms. Let $T=Y \rtimes G$, noting that $T$ forms an $E$-unitary inverse monoid with identity $\left(1, g^{0}\right)$. Hence Sect. 5.9 of [13] applies, so that $\left(1, g^{n}\right)^{-1}=\left(1, g^{-n}\right)$ and $\left(e_{q}, g^{n}\right)^{-1}=\left(g^{-n} \cdot e_{q}, g^{-n}\right)=\left(e_{q-n}, g^{-n}\right)$ for each $q \in \mathbb{Q}$ and $n \in \mathbb{Z}$. Let $W$ be the inverse subsemigroup of $S$ generated by $\left\{(1, g),\left(e_{1}, g\right)\right\}$. Then it is a simple exercise to show that

$$
W=\left\langle(1, g),\left(1, g^{-1}\right),\left(e_{1}, g\right),\left(e_{0}, g^{-1}\right)\right\rangle=\left\{\left(1, g^{n}\right),\left(e_{m}, g^{n}\right): m, n \in \mathbb{Z}\right\},
$$

with $E(W)=\left\{\left(1, g^{0}\right),\left(e_{n}, g^{0}\right): n \in \mathbb{Z}\right\}$. Let $\left(A, g^{n}\right) \in S$. If $A=e_{p}$ for some $p \in \mathbb{Q}$ then take $k \in \mathbb{Z}$ such that $n+k<p$, and if $A=1$ then take any $k \in \mathbb{Z}$. Then $\left(e_{k}, g^{-n}\right) \in W$ and

$$
\left(A, g^{n}\right)\left(e_{k}, g^{-n}\right)=\left(A e_{n+k}, g^{n-n}\right)=\left(e_{n+k}, g^{0}\right) \in E(W) .
$$

Hence $\omega_{S}^{\ell}$ is finitely generated by Corollary 5.2.

Note that $\left(A, g^{n}\right) \mathcal{J}\left(B, g^{m}\right)$ if and only if there exist $z, t \in \mathbb{Z}$ such that $g^{z} \cdot A \leq B$ and $g^{t} \cdot B \leq A$. If $A=e_{p}$ and $B=e_{q}$ then this can be satisfied for any $z, t<$ $\min \{p-q, q-p\}$, while if $A=B=1$ then this is satisfied for any $z, t$. It follows that the $\mathcal{J}$-classes of $T$ are $J=\left\{\left(1, g^{n}\right): n \in \mathbb{Z}\right\}$ and $S \backslash J$. Hence $S \backslash J$ is the unique proper ideal of $S$, and is not finitely generated. 
Note that Example 5.6 gives the desired example of a semidirect product $S \rtimes T$ with $\omega_{S \rtimes T}^{\ell}$ being finitely generated, but such that $\omega_{S}^{\ell}$ is not.

Corollary 5.7 Let $E$ be a semilattice. Then the following statements are equivalent:

(1) $\omega_{E}^{\ell}$ is finitely generated;

(2) E is pseudo-finite;

(3) $E$ has a least element and there is a finite set $U \subseteq E$ such that for every $e \in E$ we have $e \leq u$ for some $u \in U$.

Proof (1) $\Rightarrow$ (3) follows from Theorem 5.1 and the fact a finitely generated subsemigroup of a semilattice is finite. (3) $\Rightarrow(2)$ is immediate from Proposition 5.3 and (2) $\Rightarrow$ (1) follows by definition.

Corollary 5.8 [9, Theorem 9] Let E be a semilattice with identity 1. Then $\omega_{E}^{\ell}$ is finitely generated if and only if $E$ is pseudo-finite if and only if $E$ has a least element.

It follows from Theorem 5.1 and Proposition 5.3 that:

Corollary 5.9 Let $S=\mathcal{B}^{0}(G, I)$ be a Brandt semigroup over a group $G$. Then the following statements are equivalent:

(1) I is finite;

(2) $\omega_{S}^{\ell}$ is finitely generated;

(3) $S$ is pseudo-finite.

Notice that we find another approach to Corollary 5.9 in the next section when dealing with arbitrary Rees matrix semigroups.

\section{Rees matrix semigroups}

In this section we examine Rees matrix semigroups $\mathcal{M}=\mathcal{M}[S ; I, \Lambda ; P]$ and Rees matrix semigroups with zero $\mathcal{M}^{0}=\mathcal{M}^{0}[S ; I, \Lambda ; P]$ over a semigroup $S$. Note that we make no restriction on the elements of $P=\left(p_{\lambda i}\right)$. Of course, if $S=G$ is a group, then $\mathcal{M}=\mathcal{M}[G ; I, \Lambda ; P]$ is completely simple, and if every row/column of $P$ contains a non-zero entry then $\mathcal{M}^{0}=\mathcal{M}^{0}[G ; I, \Lambda ; P]$ is completely 0 -simple. We recall from [9] that completely simple semigroups of type left-FP $\mathrm{F}_{1}$ were considered, but the convention in [9] is that one considers the property for the corresponding monoid obtained by adjoining an identity.

There are four cases for us to consider that arise from the existence or otherwise of an identity, and the existence or otherwise of a zero:

(1) $T=\mathcal{M}^{0}[S ; I, \Lambda ; P]^{1}$;

(2) $T=\mathcal{M}[S ; I, \Lambda ; P]$;

(3) $T=\mathcal{M}^{0}[S ; I, \Lambda ; P]$;

(4) $T=\mathcal{M}[S ; I, \Lambda ; P]^{1}$.

We discuss each of them in turn and specialise to the case where $S$ is a group. We conclude with some remarks on the pseudo-finite case. 
The first case is trivial, because any monoid with zero is pseudo-finite by Corollary 2.15 .

We now consider the second case.

Theorem 6.1 Let $T=\mathcal{M}[S ; I, \Lambda ; P]$ be a Rees matrix semigroup over a semigroup $S$. Then $\omega_{T}^{\ell}$ is finitely generated if and only if the following conditions hold:

(1) I and $\Lambda$ are finite;

(2) there is a finite set $V \subseteq S$ such that with

$$
H=\left\{\left(p_{v i} a, p_{\nu j} b\right): v \in \Lambda, i, j \in I, a, b \in V\right\}
$$

every element of $S$ is $\rho_{H}$-related to an element of $V$.

Proof Suppose that $\omega_{T}^{\ell}=\left\langle U^{2}\right\rangle$ for some finite set $U=I^{\prime} \times V \times \Lambda^{\prime}$ where $I^{\prime}, V, \Lambda^{\prime}$ are finite subsets of $I, S$ and $\Lambda$, respectively. If $T$ is finite we can take $T=U$ and $V=S$ and we are done. Otherwise, let $(i, a, \lambda),(j, b, \mu) \in T$ be distinct. Then there exists a $U^{2}$-sequence

$$
(i, a, \lambda)=t_{1}\left(i_{1}, a_{1}, \lambda_{1}\right), t_{1}\left(j_{1}, b_{1}, \mu_{1}\right)=t_{2}\left(i_{2}, a_{2}, \lambda_{2}\right), \ldots, t_{n}\left(j_{n}, b_{n}, \mu_{n}\right)=(j, b, \mu)
$$

where $t_{m} \in T^{1}$ and $\left(\left(i_{m}, a_{m}, \lambda_{m}\right),\left(j_{m}, b_{m}, \mu_{m}\right)\right) \in U^{2}$ for all $1 \leq m \leq n$. Clearly $\lambda=\lambda_{1} \in \Lambda^{\prime}$, so that $\Lambda=\Lambda^{\prime}$ is finite. Furthermore, if $I$ is infinite, then we can pick $i$ and $j$ above as being distinct elements of $I \backslash I^{\prime}$. It is then easy to see that each $t_{i} \in T$ and reading from left to right the first co-ordinate of each $t_{k}$ is equal to $i$, which is not possible in view of the final equality. Hence $I$ is finite. On the other hand, choosing $i=j, \lambda=\mu$, and $b \in V$, then taking $a \in S$ to be arbitrary we have that $a$ is connected to an element $b^{\prime} \in V$ via an $H$-sequence. Indeed, either $t_{k} \in T$ for $1 \leq k \leq n$, in which case $b=b^{\prime}$, or letting $k$ be least with $t_{k}=1$, we have $a \rho_{H} a_{k}=b^{\prime} \in \bar{V}$.

Conversely, suppose that (1) and (2) hold. Let $W=\{a \in S:(a, b) \in$ $H$ for some $b \in S\}$. We claim that $\omega_{T}^{\ell}=\left\langle Q^{2}\right\rangle$ where $Q=I \times(V \cup W) \times \Lambda$. To see this, let $(i, a, \lambda) \in T$. If $(i, a, \lambda) \in Q$ we are done. Otherwise, $a$ is connected via an $H$-sequence

$$
a=k_{1} a_{1}, k_{1} b_{1}=k_{2} a_{2}, \ldots, k_{n} b_{n}=b
$$

where $b \in V$. Fix our notation as $\left(a_{l}, b_{l}\right)=\left(p_{\eta_{l} i_{l}} u_{l}, p_{\eta_{l} j_{l}} v_{l}\right) \in H$ for all $1 \leq l \leq n$. If every $k_{\ell} \in S$, then we have a $Q^{2}$-sequence

$$
\begin{aligned}
(i, a, \lambda) & =\left(i, k_{1}, \eta_{1}\right)\left(i_{1}, u_{1}, \lambda\right),\left(i, k_{1}, \eta_{1}\right)\left(j_{1}, v_{1}, \lambda\right) \\
& =\left(i, k_{2}, \eta_{2}\right)\left(i_{2}, u_{2}, \lambda\right), \ldots,\left(i, k_{n}, \eta_{n}\right)\left(j_{n}, v_{n}, \lambda\right)=(i, b, \lambda) .
\end{aligned}
$$

On the other hand, if $\ell$ is the least occurrence of $k_{\ell}=1$, we have a $Q^{2}$-sequence

$$
\begin{aligned}
(i, a, \lambda) & =\left(i, k_{1}, \eta_{1}\right)\left(i_{1}, u_{1}, \lambda\right),\left(i, k_{1}, \eta_{1}\right)\left(j_{1}, v_{1}, \lambda\right) \\
& =\left(i, k_{2}, \eta_{2}\right)\left(i_{2}, u_{2}, \lambda\right), \ldots,\left(i, k_{\ell-1}, \eta_{\ell-1}\right)\left(j_{\ell-1}, v_{\ell-1}, \lambda\right)=(i, c, \lambda)
\end{aligned}
$$


where $c \in W$. In either case, $(i, a, \lambda)$ is $Q^{2}$-related to an element of $Q$, completing the proof.

Consequently, for the Rees matrix semigroup $T=\mathcal{M}[S ; I, \Lambda ; P]$, if $\omega_{T}^{\ell}$ is finitely generated, then $P$ is a finite matrix and $\omega_{S}^{\ell}$ is finitely generated. The following example shows that the converse needs not be true.

Example 6.2 Let $S$ be an infinite monoid with zero, so that $\omega_{S}^{\ell}$ is finitely generated by Corollary 2.15. Let $P$ be the $1 \times 1$ matrix $P=(0)$. Then $T=\mathcal{M}[S ; 1,1 ; P]$ is an infinite null semigroup, and thus $\omega_{T}^{\ell}$ is not finitely generated.

Corollary 6.3 Let $T=\mathcal{M}[G ; I, \Lambda ; P]$ be a Rees matrix semigroup over a group $G$. Then $\omega_{T}^{\ell}$ is finitely generated if and only if $I, \Lambda$ are finite, and $G$ is finitely generated.

Proof Suppose that $\omega_{T}^{\ell}$ is finitely generated so that (1) and (2) of Theorem 6.1 hold. With the notation of that result, for any $g \in G$ we have an $H$-sequence connecting $g$ to some element $v \in V$, so that by a standard argument, $v^{-1} g \in\langle K\rangle$ and hence $g \in\langle K \cup V\rangle$ where $K=\left\{u^{-1} v:(u, v) \in H\right\}$. Hence $G$ is finitely generated.

Conversely, if $I, \Lambda$ are finite and $G$ is finitely generated by $L$, say, then with $\bar{L}=\{(1, l): l \in L\}$ we have that $\bar{L}$ generates $\omega_{G}^{\ell}$. Since $\bar{L}=\left\{\left(p p^{-1}, p p^{-1} l\right): p \in\right.$ $P, l \in L\}$ we have $L^{\prime}=\left\{\left(p_{v i} a, p_{v j} b\right): v \in \Lambda, i \in I, a, b \in Q\right\}$ generates $\omega_{G}^{l}$ where $Q=\left\{p^{-1}, p^{-1} l: p \in P, l \in L\right\}$. The result now follows from Theorem 6.1.

Since a rectangular band is isomorphic to a Rees matrix semigroup over the trivial group it follows that:

Corollary 6.4 Let $B$ be a rectangular band. Then $\omega_{B}^{\ell}$ is finitely generated if and only if $B$ is pseudo-finite if and only if $B$ is finite.

Theorem 6.5 Let $T=\mathcal{M}[S ; I, \Lambda ; P]^{1}$ be a Rees matrix semigroup over a semigroup $S$, with identity adjoined. Then $\omega_{T}^{\ell}$ is finitely generated if and only if the following conditions hold:

(1) I is finite;

(2) there is a finite set $V \subseteq S$ and a finite subset $Q$ of entries of $P=\left(p_{\lambda, i}\right)$ such that any element a of $S$ is $\rho_{U}$-related to an element of $V$ via the left congruence $\rho_{U}$ defined on $S^{1}$, where

$U=\left\{\left(p_{v i} a, p_{v j} b\right): v \in \Lambda, i, j \in I, a, b \in V\right\} \cup\{(1, p v),(p v, 1): p \in Q, v \in V\}$,

via a $U$-sequence of the form

$$
a=t_{1} c_{1}, t_{1} d_{1}=t_{2} c_{2}, \ldots, t_{k} d_{k}=v,
$$

where $t_{i} \in S$ and $\left(d_{j}, c_{j+1}\right) \neq(1,1)$ for any $1 \leq j<k$.

Proof Suppose that $\omega_{T}^{\ell}=\left\langle X^{2}\right\rangle$ for some finite set $X=\left(I^{\prime} \times V \times \Lambda^{\prime}\right) \cup\{1\}$. 
If $I$ is infinite, then we can pick $i \in I \backslash I^{\prime}$ and choosing $(i, a, \lambda) \in T$ we have $(i, a, \lambda) \rho_{X^{2}} 1$, so that there exists an $X^{2}$-sequence

$$
(i, a, \lambda)=z_{1} r_{1}, z_{1} s_{1}=z_{2} r_{2}, \ldots, z_{n} s_{n}=1
$$

where $z_{m} \in T$ and $\left(r_{m}, s_{m}\right) \in X^{2}$ for all $1 \leq m \leq n$. As $i \in I \backslash I^{\prime}$, we have $z_{1} \in\{i\} \times S \times \Lambda$, implying $z_{2}, \ldots, z_{n} \in\{i\} \times S \times \Lambda$, but $z_{n} s_{n}=1$ forces $z_{n}=1$, a contradiction.

Fix $(i, a, \mu) \in T$ where $\mu \in \Lambda^{\prime}$. We have an $X^{2}$-sequence, which we may assume to be of minimum length (hence $\left(d_{j}, c_{j+1}\right) \neq(1,1)$ for any $1 \leq j<n$ )

$$
(i, a, \mu)=t_{1} c_{1}, t_{1} d_{1}=t_{2} c_{2}, \ldots, t_{n} d_{n}=1,
$$

so that $t_{i} c_{i} \neq 1$ for $1 \leq i \leq n$. Notice that $t_{n}=1$. Suppose that $t_{1}, \ldots, t_{k} \neq 1$ and $t_{k+1}=1$ for some $k \in\{1, \ldots, n-1\}$. We therefore have a sequence

$$
a=t_{1}^{\prime} c_{1}^{\prime}, t_{1}^{\prime} d_{1}^{\prime}=t_{2}^{\prime} c_{2}^{\prime}, \ldots, t_{k}^{\prime} d_{k}^{\prime}=a_{k+1}^{\prime}
$$

where if $t_{l}=\left(g_{l}, w_{l}, \eta_{l}\right)$ we have $t_{l}^{\prime}=w_{l}$, and

$$
c_{l}^{\prime}= \begin{cases}1 & \text { if } c_{l}=1 \\ p_{\eta_{l} h_{l}} a_{l} & \text { if } c_{l}=\left(h_{l}, a_{l}, \lambda_{l}\right)\end{cases}
$$

and

$$
d_{l}^{\prime}= \begin{cases}1 & \text { if } d_{l}=1 \\ p_{\eta_{l} k_{l}} b_{l} & \text { if } d_{l}=\left(k_{l}, b_{l}, \mu_{l}\right) .\end{cases}
$$

Let $Q=\left\{p_{\nu j}: v \in \Lambda^{\prime}, j \in I\right\}$. We show by induction on the length of the sequence that for $1 \leq l \leq k$ if $c_{l}=1$ (respectively, $d_{l}=1$ ), then $t_{l} \in I \times S \times \Lambda^{\prime}$ and $d_{l}^{\prime} \in Q V$ (respectively, $c_{l}^{\prime} \in Q V$ ).

Starting with $l=1$, if $c_{1}=1\left(\operatorname{so} d_{1} \neq 1\right)$ then $t_{1}=(i, a, \mu)=\left(g_{1}, w_{1}, \eta_{1}\right)$ and $d_{1}^{\prime}=p_{\eta_{1} k_{1}} b_{1}=p_{\mu k_{1}} b \in Q V$. On the other hand, if $d_{1}=1$ (so $c_{1} \neq 1$ ) then, noticing that $c_{2} \neq 1$ (else our sequence could be replaced by a shorter one) we have $t_{1}=t_{2} c_{2}$ so that $\eta_{1} \in \Lambda^{\prime}$ and $c_{1}=p_{\eta_{1} h_{1}} a_{1} \in Q V$. Suppose for induction that $2 \leq m \leq k$ and the result holds for all sequences of length strictly less than $m$, and let our sequence be of length $m$. Consider $t_{m-1} d_{m-1}=t_{m} c_{m}$ which, by our inductive assumption, must lie in $I \times S \times \Lambda^{\prime}$. If $c_{m}=1\left(\right.$ so $\left.d_{m} \neq 1\right)$ then $t_{m}=t_{m-1} d_{m-1} \in I \times S \times \Lambda^{\prime}$ and $d_{m}^{\prime}=p_{\eta_{m} k_{m}} b_{m} \in Q V$. On the other hand, if $d_{m}=1$ (so $c_{m} \neq 1$ ), then $c_{m+1} \neq 1$, so that $t_{m}=t_{m+1} c_{m+1} \in I \times S \times \Lambda^{\prime}$ and $c_{m}^{\prime}=p_{\eta_{m} h_{m}} a_{m} \in Q V$. Thus (2) holds, with $U$ as defined.

Suppose for the converse that (1) and (2) hold. Let $\Lambda^{\prime}$ be a finite subset of $\Lambda$ chosen such that $Q \subseteq V=\left\{p_{\lambda i}: \lambda \in \Lambda^{\prime}, i \in I\right\}$. Let

$$
W=\left(I \times V \times \Lambda^{\prime}\right) \cup\{1\}
$$


We show that $\omega_{T}^{\ell}=\left\langle W^{2}\right\rangle$.

Let $(i, a, \lambda) \in T$ and pick any $b \in V, \mu \in \Lambda^{\prime}$. Let $s=a p_{\lambda i} b$. Notice that

$$
(i, a, \lambda)=(i, a, \lambda) 1 \rho_{W^{2}}(i, a, \lambda)(i, b, \mu)=(i, s, \mu) .
$$

By assumption, there is a $U$-sequence in $S^{1}$

$$
s=t_{1}^{\prime} c_{1}^{\prime}, t_{1}^{\prime} d_{1}^{\prime}=t_{2}^{\prime} c_{2}^{\prime}, \ldots, t_{n}^{\prime} d_{n}^{\prime}=v
$$

for some $v \in V$, where no $t_{l}^{\prime}=1$ and no pair $\left(c_{l}^{\prime}, d_{l+1}^{\prime}\right)=(1,1)$. We aim to 'lift' this sequence to a $W^{2}$-sequence

$$
(i, s, \kappa)=t_{1} c_{1}, t_{1} d_{1}=t_{2} c_{2}, \ldots, t_{n} d_{n}=(i, v, \tau)
$$

in $T^{1}$, for some $\kappa, \tau \in \Lambda^{\prime}$. For each $1 \leq l \leq n$ we have $\left(c_{l}^{\prime}, d_{l}^{\prime}\right)=\left(p_{\eta_{l} h_{l}} a_{l}, p_{\eta_{l} k_{l}} b_{l}\right)$, or $\left(c_{l}^{\prime}, d_{l}^{\prime}\right)=\left(1, p_{\eta_{l} k_{l}} b_{l}\right)$ or $\left(c_{l}^{\prime}, d_{l}^{\prime}\right)=\left(p_{\eta_{l} h_{l}} a_{l}, 1\right)$. Fix $\mu \in \Lambda^{\prime}$, put $t_{l}=\left(i, t_{l}^{\prime}, \eta_{l}\right)$ for $1 \leq l \leq n$ and define

$$
c_{j}= \begin{cases}1 & \text { if } c_{j}^{\prime}=1 \\ \left(h_{j}, a_{j}, \eta_{j-1}\right) & \text { if } d_{j-1}=1 \\ \left(h_{j}, a_{j}, \mu\right) & \text { otherwise }\end{cases}
$$

and

$$
d_{j}= \begin{cases}1 & \text { if } d_{j}^{\prime}=1 \\ \left(k_{j}, b_{j}, \eta_{j+1}\right) & \text { if } c_{j+1}=1 \\ \left(k_{j}, b_{j}, \mu\right) & \text { otherwise. }\end{cases}
$$

It is then clear that with $\kappa=\eta_{1}$ if $c_{1}^{\prime}=1$, and $\kappa=\mu$ else, and $\tau=\eta_{n}$ if $d_{n}=1$ and $\tau=\mu$ else, we have a $W^{2}$-sequence connecting $(i, s, \kappa)$ to $(i, v, \tau)$. Since $(i, a, \lambda) \rho_{W^{2}}(i, s, \kappa)$ and $(i, v, \tau) \in W$, we are done.

Corollary 6.6 Let $B$ be a rectangular band. Then $\omega_{B^{1}}^{\ell}$ is finitely generated if and only if $B^{1}$ is pseudo-finite if and only if $B$ has finitely many $\mathcal{R}$-classes.

Proof We need only consider the issue of pseudo-finiteness. If $B$ has finitely many $\mathcal{R}$ classes $\left\{R_{a_{i}}: 1 \leq i \leq n\right\}$, then letting $X=\left\{1, a_{i}: 1 \leq i \leq n\right\}$, for any $b \in B$ we have $b \in R_{a_{i}}$ for some $i$ and then $b=b 1 \rho_{X^{2}} b a_{i}=a_{i} \rho_{X^{2}} 1$, so that $B$ is pseudo-finite.

In the case where $S$ is a monoid in the hypothesis of Theorem 6.5 we obtain little simplification beyond the obvious ability to drop the condition that $t_{i} \in S$; the condition on the pair $\left(d_{j}, c_{j+1}\right)$ is redundant, as it merely says the sequence cannot be reduced by setting $t_{j} c_{j}=t_{j+1} d_{j+1}$. Where $S$ is a group we may simplify considerably.

Suppose $S$ has been normalised in row 1 and column 1 , that is, there exists $1 \in I \cap \Lambda$ such that $p_{1 i}=p_{\lambda 1}=1$ for all $i \in I$ and $\lambda \in \Lambda$. Let $G^{P}=\left\{p_{\lambda i}: i \in I, \lambda \in \Lambda\right\}$. By [12],

$$
\langle E(S)\rangle=\mathcal{M}\left[\left\langle G^{P}\right\rangle ; I, \Lambda ; P\right] .
$$


Corollary 6.7 Let $T=\mathcal{M}[G ; I, \Lambda ; P]^{1}$ be a normalised Rees matrix semigroup over a group $G$. Then $\omega_{T}^{\ell}$ is finitely generated if and only if the following conditions hold:

(1) I is finite;

(2) $G=\left\langle G^{P} \cup V\right\rangle$ where $V$ is a finite set.

Proof Suppose that $\omega_{T}^{\ell}$ is finitely generated. Thus Theorem 6.5 holds and there are finite sets $V \subseteq G, Q \subseteq G^{P}$, and $U \subseteq G \times G$ as in (2) of that result. Augment $V$ by $\left\{q^{-1}: q \in Q\right\}$ and, noticing that $(1, q v)=\left(q q^{-1}, q v\right)$ and $(q v, 1)=\left(q v, q q^{-1}\right)$ we have that

$$
U=\left\{\left(p_{v i} a, p_{v j} b\right): v \in \Lambda, i, j \in I, a, b \in V\right\} .
$$

Hence by previous remarks we have that

$$
G=\left\langle\left\{p_{\nu i} a b^{-1} p_{\nu j}^{-1}: a, b \in V, v \in \Lambda, i, j \in I\right\}\right\rangle=\left\langle G^{P} \cup V\right\rangle,
$$

noting that $1 \in I$, and so (2) holds.

Conversely, if (1) and (2) hold then it is easy to see that $\omega_{G}^{l}$ has a set of generators of the form

$$
\left\{\left(p_{v i}, p_{v j}\right): v \in \Lambda, i, j \in I\right\} \cup\left\{(1, v),\left(1, v^{-1}\right): v \in V\right\}
$$

and hence of the form

$$
\left\{\left(p_{v i}, p_{v j}\right): v \in \Lambda, i, j \in I\right\} \cup\left\{\left(p_{\tau k} p_{\tau k}^{-1}, p_{\tau k} p_{\tau k}^{-1} v\right),\left(p_{\tau k} p_{\tau k}^{-1}, p_{\tau k} p_{\tau k}^{-1} v^{-1}\right): v \in V\right\}
$$

where $p_{\tau k}$ is chosen and fixed, and hence (by suitably augmenting $V$ to give a finite set $W$ ) a set of generators of the form

$$
\left\{\left(p_{\nu i} a, p_{\nu j} b\right): v \in \Lambda, i, j \in I, a, b \in W\right\}
$$

In view of earlier comments, the result now follows from Theorem 6.5.

Recall that in [9], a semigroup is said to be of type left-FP 1 if the monoid $S^{1}$ has this property. In light of (6.1) we may now recover Theorems 4 and 7 of [9].

Corollary 6.8 Let $T=U^{1}$ be a completely simple semigroup with an identity adjoined and let $G$ be a maximal subgroup of $U$. Then $\omega_{T}^{\ell}$ is finitely generated if and only if $U$ has finitely many $\mathcal{R}$-classes and $G$ is generated by a set $\langle(\langle E(S)\rangle \cap G) \cup V\rangle$ where $V$ is finite. If in addition $U$ has finitely many $\mathcal{L}$-classes, then $\omega_{T}^{\ell}$ is finitely generated if and only if $U$ has finitely many $\mathcal{R}$-classes and $G$ is finitely generated.

Finally we consider the case when $T=\mathcal{M}^{0}[S ; I, \Lambda ; P]$. 
Proposition 6.9 Let $T=\mathcal{M}^{0}[S ; I, \Lambda ; P]$ be a Rees matrix semigroup with zero over a semigroup $S$. Then $\omega_{T}^{\ell}$ is finitely generated if and only if:

(1) $\Lambda$ is finite; and

(2) (a) I is finite and there is a finite set $V \subseteq S$ such that for every a $\in S \backslash V$, we have $S^{1} a \subseteq S p_{v i} v$ for some $v \in \Lambda, i \in I$ and $v \in V$, or

(b) $I$ is infinite and there is a finite set $V \subseteq S$ and a finite set $I^{\prime} \subseteq I$ such that for every $a \in S$, we have $S^{1} a \subseteq S p_{v i} v$ for some $v \in \Lambda, i \in I^{\prime}$ and $v \in V$.

Proof Suppose that $\omega_{T}^{\ell}=\left\langle U^{2}\right\rangle$ for some finite set $U=\left(I^{\prime} \times V \times \Lambda^{\prime}\right) \cup\{0\}$. If $T$ is finite then (1) and (2) (a) trivially hold. Otherwise, we can find distinct $(i, a, \lambda),(j, b, \mu) \notin$ $U$. By assumption, there exists a $U^{2}$-sequence

$$
(i, a, \lambda)=t_{1} w_{1}, t_{1} q_{1}=t_{2} w_{2}, \ldots, t_{n} q_{n}=(j, b, \mu)
$$

where $t_{m} \in T^{1}$ and $\left(w_{m}, q_{m}\right) \in U^{2}$ for all $1 \leq m \leq n$. Notice that $w_{1}$ must be some $\left(i_{1}, a_{1}, \lambda_{1}\right) \in U \backslash\{0\}$, implying $\lambda=\lambda_{1} \in \Lambda^{\prime}$, so that $\Lambda=\Lambda^{\prime}$ is finite and (1) holds.

Suppose that $I$ is finite. For any $a \notin V$ we have $(i, a, \lambda) \notin U$ and then (since $T$ is not finite) we can begin a sequence as above, yielding $t_{1}=(i, c, v) \in T$. We then have that $a=c p_{v i_{1}} a_{1}$, giving $S^{1} a \subseteq S p_{v i_{1}} v$ as required, and so (2)(a) holds. On the other hand, if $I$ is infinite then for any $a \in S$ we can find an element $(i, a, \lambda) \notin U$ and a similar argument shows that (2)(b) holds.

Conversely, suppose that (1), and (2)(a) or (2)(b) hold. If $T$ is finite we are done. Otherwise, let $U=\{0\} \cup\left\{(i, v, \lambda): i \in I^{\prime}, v \in V, \lambda \in \Lambda\right\}$, where in case (2)(a) we take $I^{\prime}=I$. We show that $\omega_{T}^{\ell}=\left\langle U^{2}\right\rangle$. To see this, let $(i, a, \lambda) \in T$. If $(i, a, \lambda) \in U$ we are done. Otherwise, in case (2)(a), since $a \notin V$ we have $a=x p_{v j} v$ for some $v \in \Lambda, j \in I^{\prime}, v \in V$ and $x \in S$, giving

$$
(i, a, \lambda)=(i, x, v)(j, v, \lambda) \rho_{U^{2}}(i, x, v) 0=0 .
$$

In case (2)(b) we can write any $a \in S$ as $a=x p_{v j} v$ for some $v \in \Lambda, j \in I^{\prime}, v \in V$ and $x \in S$, and achieve our aim.

Again we may simplify the proposition above for groups.

Corollary 6.10 Let $T=\mathcal{M}^{0}[G ; I, \Lambda ; P]$ be a Rees matrix semigroup with zero over a group $G$. Then $\omega_{T}^{\ell}$ is finitely generated if and only if either $T$ is finite, or $\Lambda$ is finite and $T$ is non-null.

Proof Recall that $T$ is non-null if and only if the sandwich matrix has a non-zero element.

Remark 6.11 We end by considering again our four cases of whether or not our Rees matrix semigroup has a zero or 1 in the context of being pseudo-finite. In cases (1) and (3) we have a semigroup with zero, hence by Corollary 2.14, the universal relation of our semigroup is finitely generated if and only if it is pseudo-finite. In Theorems 6.1 and 6.5 and their corollaries we merely need to impose a bound on the length of the sequences to achieve the criterion for being pseudo-finite (so that in Corollaries 6.3 and 6.8, the group $G$ is required to be finite). 
Open Question 6.12 Let $T=\mathcal{M}[S ; I, \Lambda ; P]$. Fixing $(i, \lambda) \in I \times \Lambda$ we have that $K_{i \lambda}=\{(i, s, \lambda): s \in S\}$ becomes a variant of $S$ with sandwich element $p_{\lambda i}$. In view of the results of this section we ask, what is the connection between $\omega_{S}^{\ell}$ being finitely generated and $\omega_{S^{a}}^{\ell}$ being finitely generated, for a variant $S^{a}$ of $S$ ?

\section{Strong semilattices of semigroups and Bruck-Reilly extensions}

Strong semilattices of monoids which are of type left-FP ${ }_{n}$, and hence of type left-FP 1 , have been classified in [9, Theorem 9]. Our aim in this section is to generalise their result, by studying strong semilattices of semigroups, with and without an identity adjoined, and to examine the property of being pseudo-finite in this context. Using a strong semilattice of groups we are able to give a counterexample to the conjecture of Dales and White, mentioned in the introduction. We also examine Bruck-Reilly extensions $\mathrm{BR}=\mathrm{BR}(S, \theta)$ of a monoid $S$ to determine when $\omega_{\mathrm{BR}}^{\ell}$ is finitely generated. For details concerning strong semilattices of semigroups, and Bruck-Reilly extensions, we refer the reader to [13]. Note that in the result below, even if our component semigroups are monoids, we are not assuming that the connecting morphisms $\varphi_{\alpha, \beta}$ are monoid morphisms.

Proposition 7.1 Let $S=\left[\mathcal{Y} ; S_{\alpha} ; \varphi_{\alpha, \beta}\right]$ be a strong semilattice of semigroups. Then $\omega_{S}^{\ell}$ is finitely generated ( $S$ is pseudo-finite) if and only if

(1) there exists a finite subset $X$ of $S$ such that for every $a \in S$ we have some $x \in X$ with $a \leq_{\mathcal{L}} x$;

(2) $\mathcal{Y}$ has a least element 0 ;

(3) $\omega_{S_{0}}^{\ell}$ is finitely generated ( $S_{0}$ is pseudo-finite).

Proof Suppose that $\omega_{S}^{\ell}=\left\langle X^{2}\right\rangle$ for some finite set $X \subseteq S$. Then (1) holds by Lemma 2.9. Since there is a natural epimorphism from $S$ to $\mathcal{Y}$, we deduce that $\omega_{\mathcal{Y}}^{\ell}$ is finitely generated by Proposition 4.1, so that $\mathcal{Y}$ has a least element by Corollary 5.7. Further, $S_{0}$ is a morphic image of $S$ under $\psi: S \rightarrow S_{0}$ defined by $s \psi=s \varphi_{\alpha, 0}$ for $s \in S_{\alpha}$. Thus $\omega_{S_{0}}^{\ell}$ is finitely generated; if $S$ is pseudo-finite, then $S_{0}$ is also pseudo-finite.

Conversely, suppose (1)-(3) hold. Then $S_{0}$ forms an ideal of $S$, and so the result follows from Corollary 2.13.

Proposition 7.2 Let $S=\left[\mathcal{Y} ; S_{\alpha} ; \varphi_{\alpha, \beta}\right]$ be a strong semilattice of semigroups that is not a monoid. Then $\omega_{S^{1}}^{\ell}$ is finitely generated ( $S^{1}$ is pseudo-finite) if and only if $\mathcal{Y}$ has a least element 0 and $\omega_{S_{0}^{\ell}}^{\ell}$ is finitely generated ( $S_{0}^{1}$ is pseudo-finite).

Proof Suppose that $\omega_{S^{1}}^{\ell}$ is finitely generated ( $S^{1}$ is pseudo-finite). Since there is a natural epimorphism from $S^{1}$ to $\mathcal{Y}^{1}$, we deduce that $\omega_{\mathcal{Y}^{1}}^{\ell}$ is finitely generated, so that $\mathcal{Y}^{1}$ (and so $\mathcal{Y}$ ) has a least element 0 by Corollary 5.8. For convenience denote the identity of $S^{1}$ by $1_{S}$ and the identity of $S_{0}^{1}$ by $1_{S_{0}}$. Also, $S_{0}^{1}$ is a morphic image of $S^{1}$ under $\psi: S^{1} \rightarrow S_{0}^{1}$ defined by $s \psi=s \varphi_{\alpha, 0}$ for $s \in S_{\alpha}$ and $1_{S} \psi=1_{S_{0}}$. Thus $\omega_{S_{0}^{\ell}}^{\ell}$ is finitely generated ( $S_{0}^{1}$ is pseudo-finite) by Proposition 4.1 . 
Conversely, suppose that $\mathcal{Y}$ has a least element 0 and $\omega_{S_{0}^{1}}^{\ell}=\left\langle X^{2}\right\rangle$ for a finite set $X \subseteq S_{0}^{1}$. Fix $u \in X \cap S_{0}$ and let $Y=\left(X \cap S_{0}\right) \cup\left\{1_{S}\right\}$. If $t \in S_{0}$ then as $t \rho_{X^{2}} u$ in $S_{0}^{1}$, it is easy to see, replacing $1_{S_{0}}$ by $1_{S}$ if necessary, that $t \rho_{Y^{2}} u$ in $S^{1}$ via a $Y^{2}$-sequence of the same length as the original $X^{2}$-sequence. For any $s \in S$ we have $s u \in S_{0}$, giving $s=s 1_{S} \rho_{Y^{2}}$ su $\rho_{Y^{2}} u \rho_{Y^{2}} 1_{S}$, so that $\rho_{Y^{2}}=\omega_{S^{1}}^{\ell}$. The statement on pseudo-finiteness follows.

Corollary 7.3 cf. [9, Corollary 3] Let $S=\left[\mathcal{Y} ; G_{\alpha} ; \varphi_{\alpha, \beta}\right]$ be a Clifford monoid. Then $\omega_{S}^{\ell}$ is finitely generated ( $S$ is pseudo-finite) if and only if $\mathcal{Y}$ has a least element 0 and $G_{0}$ is finitely generated (finite).

Remark 7.4 We recall that a normal band is a strong semilattice of rectangular bands. We may immediately apply Propositions 7.1 and 7.2 to this case, calling upon Corollaries 6.4 and 6.6. We examine bands in greater detail in Sect. 8 .

Our aim now is to extend Example 5.5 by considering arbitrary Bruck-Reilly extensions of monoids:

Proposition 7.5 Let $S$ be a monoid with $\omega_{S}^{\ell}$ being finitely generated and let $T=$ $B R(S, \theta)$ be the Bruck-Reilly extension of $S$ determined by $\theta$. Then $\omega_{T}^{\ell}$ is finitely generated, but $T$ is not pseudo-finite.

Proof Let $e$ be the identity of $S$ and $\omega_{S}^{\ell}=\left\langle X^{2}\right\rangle$ for a finite set $X \subseteq S$. Let $Y=$ $\{(1, e, 0),(0, e, 0),(0, x, 0): x \in X\}$. Notice that, for any $(u, a, 0) \in T$,

$$
(u, a, 0)=\underbrace{(1, e, 0) \cdots(1, e, 0)}_{u-1 \text { times }}(1, a, 0) .
$$

As $\omega_{S}^{\ell}=\left\langle X^{2}\right\rangle$, we have that $a=t_{1} c_{1}, t_{1} d_{1}=t_{2} c_{2}, \ldots, t_{n} d_{n}=e$ where $t_{i} \in$ $S,\left(c_{i}, d_{i}\right) \in X^{2}$ for all $1 \leq i \leq n$. This gives

$$
\begin{aligned}
(1, a, 0)= & \left(1, t_{1}, 0\right)\left(0, c_{1}, 0\right),\left(1, t_{1}, 0\right)\left(0, d_{1}, 0\right)=\left(1, t_{2}, 0\right)\left(0, c_{2}, 0\right), \ldots \\
& \cdots\left(1, t_{n}, 0\right)\left(0, d_{n}, 0\right)=(1, e, 0)
\end{aligned}
$$

and hence $(1, a, 0) \rho_{Y^{2}}(1, e, 0) \rho_{Y^{2}}(0, e, 0)$. Notice that $(1, a, 0) \rho_{Y^{2}}(0, e, 0)$ gives

$$
(2, a, 0)=(1, e, 0)(1, a, 0) \rho_{Y^{2}}(1, e, 0)(0, e, 0)=(1, e, 0) \rho_{Y^{2}}(0, e, 0) .
$$

By induction we derive

$$
(u, a, 0)=\underbrace{(1, e, 0) \cdots(1, e, 0)}_{u-1 \text { times }}(1, a, 0) \rho_{Y^{2}}(0, e, 0) .
$$

For any $(u, a, v) \in T$ where $v \neq 0$,

$$
(u, a, v-1)=(u, a, v)(1, e, 0) \rho_{Y^{2}}(u, a, v)(0, e, 0)=(u, a, v) .
$$


Again, a simple inductive argument yields $(u, a, 0) \rho_{Y^{2}}(u, a, v)$, so $(u, a, v) \rho_{Y^{2}}$ $(0, e, 0)$, and hence $\rho_{Y^{2}}=\omega_{T}^{\ell}$.

There exists an epimorphism from $T$ onto the Bicyclic monoid given by $(m, g, n) \psi=(m, n)$, and so the final statement is immediate from Proposition 4.1 and Example 5.5.

In the opposite direction to that in Proposition 7.5, we see that the property of $\omega^{\ell}$ being finitely generated for $\operatorname{BR}(S, \theta)$ does not necessarily transfer to $S$.

Example 7.6 Let $G$ be the free group on $\left\{a_{i}: i \in \mathbb{N}^{0}\right\}$ and let $\theta: G \rightarrow G$ be given by $a_{i} \theta=a_{i+1}$. Let $T=B R(G, \theta)$ be the Bruck-Reilly extension of $G$ determined by $\theta$. Clearly $G$ is not finitely generated. We claim that $\omega_{T}^{\ell}=\left\langle X^{2}\right\rangle$ for the finite set

$$
X=\left\{(0, e, 0),(0, e, 1),\left(1, a_{0}, 0\right),(1, e, 0)\right\} .
$$

Proof By the usual arguments, we have $(i, e, j) \rho_{X^{2}}(0, e, 0)$, for any $i, j \in \mathbb{N}^{0}$. Noting that $\left(1, a_{0}, 0\right) \in X$, we assume that $\left(0, a_{i}, 0\right) \rho_{X^{2}}(0, e, 0)$ for all $i \leq n$. Then

$$
\left(0, a_{n}, 0\right)(1, e, 0) \rho_{X^{2}}\left(0, a_{n}, 0\right)(0, e, 0)=\left(0, a_{n}, 0\right) \rho_{X^{2}}(0, e, 0) .
$$

Now

$$
\left(0, a_{n}, 0\right)(1, e, 0)=\left(1, a_{n} \theta, 0\right)=\left(1, a_{n+1}, 0\right) .
$$

From the above we have

$$
\left(0, a_{n+1}, 0\right)=(0, e, 1)\left(1, a_{n+1}, 0\right) \rho_{X^{2}}(0, e, 1)(0, e, 0)=(0, e, 1) \rho_{X^{2}}(0, e, 0),
$$

completing our inductive step. We then have

$$
(0, e, 0)=\left(0, a_{n}^{-1}, 0\right)\left(0, a_{n}, 0\right) \rho_{X^{2}}\left(0, a_{n}^{-1}, 0\right)
$$

for any $n \in \mathbb{N}^{0}$, and since the $\rho_{X^{2}}$-class of $(0, e, 0)$ is a submonoid we obtain that $(0, g, 0) \rho_{X^{2}}(0, e, 0)$ for all $g \in G$.

For any $u \in \mathbb{N}^{0}, g \in G$ we have

$$
(u, g, 0)=(1, e, 0)^{u}(0, g, 0),
$$

so that $(u, g, 0) \rho_{X^{2}}(0, e, 0)$ and then similarly to the proof of Proposition 7.5, we show via induction that $(u, g, v) \rho_{X^{2}}(0, e, 0)$, for any $v \in \mathbb{N}^{0}$.

Every monoid with zero is pseudo-finite, as is every finite monoid and hence from Proposition 4.2, every direct product of such. Dales and White [6], see also [24], posed the question of whether the converse is true, namely, that every pseudo-finite monoid is isomorphic to a direct product of a monoid with zero by a finite monoid. We answer this question negatively by the following example. 
Example 7.7 Let $\mathcal{Y}=\{\alpha, \beta\}$ be a semilattice with $\beta<\alpha$ and let $M=\left[\mathcal{Y} ; G_{\alpha} ; \varphi_{\alpha, \beta}\right]$ be a strong semilattice of groups, where $G_{\alpha}=G$ is an infinite group with identity 1 and no elements of order $2, G_{\beta}=\{a, e\}$ is a group with identity $e$, and $\varphi_{\alpha, \beta}: G_{\alpha} \rightarrow G_{\beta}$ is defined by $g \varphi_{\alpha, \beta}=e$ for all $g \in G$. Then $M$ is a pseudo-finite monoid that is not isomorphic to a direct product of a monoid with zero by a finite monoid.

Proof Clearly, $M$ does not have zero, but as $\beta$ is the least element of $\mathcal{Y}$, it follows from Corollary 7.3 that $M$ is pseudo-finite. Suppose that $U$ and $V$ are monoids and $\psi: M \rightarrow$ $U \times V$ is an isomorphism. As $|E(M)|=2$ and $|E(U \times V)|=|E(U)| \times|E(V)|$, without loss of generality, we assume $|E(U)|=2$ and $|E(V)|=1$. Let $E(U)=\left\{1_{U}, f\right\}$ and $E(V)=\left\{1_{V}\right\}$ where $1_{U}$ and $1_{V}$ are the identities of $U$ and $V$, respectively. Then $1 \psi=\left(1_{U}, 1_{V}\right)$ and $e \psi=\left(f, 1_{V}\right)$. For any $w=(p, q) \in U \times V$, we have $w \psi^{-1} \mathcal{H} 1$ or $w \psi^{-1} \mathcal{H} e$, so that $w \mathcal{H}\left(1_{U}, 1_{V}\right)$ or $w \mathcal{H}\left(f, 1_{V}\right)$, which implies $p \mathcal{H} 1_{U}, q \mathcal{H} 1_{V}$ or $p \mathcal{H} f, q \mathcal{H} 1_{V}$. Hence we deduce that $V=H_{1_{V}}$ is a group and $H_{f} \times H_{1_{V}} \cong G_{\beta}=\{a, e\}$, so that $\left|H_{f}\right|=1$ or $\left|H_{1_{V}}\right|=1$. If $\left|H_{1_{V}}\right|=|V|=1$, then $M \cong U$. If $\left|H_{f}\right|=1$ and $|V|=\left|H_{1_{V}}\right|=2$, then $V=\left\{1_{V}, b\right\}$ where $b^{2}=1_{V}$, so that the order of $\left(1_{U}, b\right)$ is 2 , so is the element $\left(1_{U}, b\right) \psi^{-1}$ in $G$, but $G$ has no elements of order 2 , a contradiction.

\section{Semigroups with a minimum ideal and completely regular semigroups}

In many of our examples of a semigroup $S$ with $\omega_{S}^{\ell}$ being finitely generated, $S$ is required to possess a minimum ideal $I$ such that $\omega_{I}^{\ell}$ is finitely generated. Clifford semigroups and normal bands illustrate this point. Indeed, if $S=\left[\mathcal{Y} ; S_{\alpha} ; \varphi_{\alpha, \beta}\right]$ is a strong semilattice of semigroups, and $\mathcal{Y}$ has a least element 0 , then any minimum ideal is contained in $S_{0}$, and if $S$ is Clifford or a normal band, $S_{0}$ will be the minimum ideal exactly. In this section we give necessary and sufficient conditions for a semigroup with a completely simple minimum ideal to have $\omega^{\ell}$ finitely generated. We apply our result to completely regular semigroups and bands. We remark that we make no restriction here on the number of right ideals of the minimum ideal, unlike [9, Theorem 8].

Theorem 8.1 Let $S$ be a semigroup with a minimum ideal $S_{0}$ that is completely simple. Then $\omega_{S}^{\ell}$ is finitely generated if and only if the following hold:

(1) there exists a finite subset $X$ of $S$ such that for every $a \in S$ we have some $x \in X$ with $a \leq_{\mathcal{L}} x$;

(2) if $G$ is a maximal subgroup of $S_{0}$, then $G=\langle F \cup V\rangle$ where $V$ is finite and $F=\left\langle E\left(C_{0}\right)\right\rangle \cap G$ where $C_{0}$ is the union of finitely many $\mathcal{R}$-classes of $S_{0}$;

(3) there exists an $\mathcal{L}$-class $L$ of $S_{0}$ and a finite subset $W \subseteq L$ such that every idempotent in $L$ is $\rho_{W^{2}}$-related to an element of $W$ via the left congruence $\rho_{W^{2}}$ defined on $S$.

Proof We first suppose that $\omega_{S}^{\ell}=\left\langle U^{2}\right\rangle$ for a finite set $U \subseteq S$. Condition (1) holds by Lemma 2.15. 
Let $H$ be a maximal subgroup in $S_{0}$ and let $e$ be identity of $H$. Let $h \in H$. As $\omega_{S}^{\ell}=\left\langle U^{2}\right\rangle$, we have

$$
h=t_{1} c_{1}, t_{1} d_{1}=t_{2} c_{2}, \ldots, t_{n} d_{n}=e,
$$

where $t_{i} \in S^{1}$ and $\left(c_{i}, d_{i}\right) \in U^{2}$. Multiplying the above sequence by $e$ from both sides, we have

$$
h=e t_{1} c_{1} e, e t_{1} d_{1} e=e t_{2} c_{2} e, \ldots, e t_{n} d_{n} e=e .
$$

Now $e t_{i} \in S_{0}$ and $\left(c_{i} e, d_{i} e\right) \in U e \times U e \subseteq S_{0} \times S_{0}$. It is convenient at this point to assume that $S_{0}$ is a Rees matrix semigroup $\mathcal{M}=\mathcal{M}[G ; I, \Lambda ; P]$ and that $H=H_{11}$ for some distinguished $1 \in I \cap \Lambda$, where $P$ is normalised in row 1 and column 1 . We can then write the above sequence as

$$
\begin{aligned}
h= & (1, g, 1)=\left(1, t_{1}^{\prime}, v_{1}\right)\left(i_{1}, c_{1}^{\prime}, 1\right),\left(1, t_{1}^{\prime}, v_{1}\right)\left(j_{1}, d_{1}^{\prime}, 1\right)=\left(1, t_{2}^{\prime}, v_{2}\right)\left(i_{2}, c_{2}^{\prime}, 1\right), \\
& \ldots,\left(1, t_{n}^{\prime}, v_{n}\right)\left(j_{n}, d_{n}^{\prime}, 1\right)=(1, f, 1)=e,
\end{aligned}
$$

where $f$ is the identity of $G,\left(1, t_{k}^{\prime}, v_{k}\right) \in e \mathcal{M}$ and $\left(\left(i_{k}, c_{k}^{\prime}, 1\right),\left(j_{k}, d_{k}^{\prime}, 1\right)\right) \in U e \times U e$. We now have

$$
g=t_{1}^{\prime} p_{v_{1} i_{1}} c_{1}^{\prime}, t_{1}^{\prime} p_{v_{1} j_{1}} d_{1}^{\prime}=t_{2}^{\prime} p_{v_{2} i_{2}} c_{2}^{\prime}, \ldots, t_{n}^{\prime} p_{v_{n} j_{n}} d_{n}^{\prime}=f
$$

which gives us $g=d_{n}^{\prime-1} p_{v_{n} j_{n}}^{-1} p_{v_{n} i_{n}} c_{n}^{\prime} d_{n-1}^{\prime-1} \cdots p_{\nu_{1} j_{1}}^{-1} p_{v_{1} i_{1}} c_{1}^{\prime}$. Let $I^{\prime}$ be the union of $\{1\}$ together with the rows indexed by the first co-ordinates of the elements of $U e$ and let $C_{0}=\mathcal{M}\left[G ; I^{\prime}, \Lambda ; P^{\prime}\right]$, where $P^{\prime}=\left(p_{\lambda i}\right)$ is the $\Lambda \times I^{\prime}$ submatrix of $P$. By (6.1), we have that $H$ is generated by $\left(\left\langle E\left(C_{0}\right)\right\rangle \cap H\right) \cup V$ where $V=U e$, and so (2) holds.

Note that $U e$ is contained in the $\mathcal{L}$-class $L_{e}$ of $S_{0}$. If $f \in E\left(L_{e}\right)$ then there exists $c_{i}, d_{i} \in U$ and $t_{i} \in S^{1}(1 \leq i \leq n)$, such that

$$
f=t_{1} c_{1}, t_{1} d_{1}=t_{2} c_{2}, \ldots, t_{n} d_{n}=e .
$$

Multiplying through by $e$ on the right, and noting that $f e=f$ we obtain (3) by augmenting $U$ by the finite set $U e$.

Conversely, suppose (1)-(3) hold. From Corollary 6.8 we know that $\omega_{C_{0}^{1}}^{\ell}$ is finitely generated, say by a set $U^{2}$. Let $G$ be a maximal subgroup in $L$ with identity $e$ and let $F=U e$. Then $F$ is a finite subset of $C_{0}$, and as any two elements of $G$ are related via a $U^{2}$-sequence in $C_{0}^{1}$, a now familiar argument shows that they are related in $S$ by an $F^{2}$-sequence of the same length.

Let $Y=X \cup W \cup F \cup\{e\}$. We claim $\omega_{S}^{\ell}=\left\langle Y^{2}\right\rangle$. Let $s \in S$ be such that $s \mathcal{L} e$, and suppose $s \mathcal{H} f=f^{2}$. Then es $\mathcal{H}$ e, so that es $\rho_{F^{2}} e$, and so es $\rho_{Y^{2}} e$. Hence fes $\rho_{Y^{2}} f e$, and so $f e s=f s=s \rho_{Y^{2}} f=f e$. However, $f \rho_{W^{2}} e$, so that $f \rho_{Y^{2}} e$, and thus $s \rho_{Y^{2}} e$. Now take any $u \in S$, and let $t \in X$ be such that $u=a t$ for some $a \in S^{1}$. Then $u=$ at $\rho_{Y^{2}}$ ae $\rho_{Y^{2}} e$ as ae $\mathcal{L} e$. Hence $\omega_{S}^{\ell}=\left\langle Y^{2}\right\rangle$. 
If we let the minimum ideal $S_{0}$ have finitely many $\mathcal{R}$-classes, then the result above simplifies as follows. Note that this corollary can be seen as an extension of Theorem 8 of [9] from monoids to semigroups.

Corollary 8.2 Let $S$ be a semigroup with a minimum ideal $S_{0}$ that is completely simple and has finitely many $\mathcal{R}$-classes. Then $\omega_{S}^{\ell}$ is finitely generated if and only if the following hold:

(1) there exists a finite subset $X$ of $S$ such that for every $a \in S$ we have some $x \in X$ with $a \leq \mathcal{L} x$

(2) if $G$ is a maximal subgroup of $S_{0}$, then $G=\langle F \cup V\rangle$ where $V$ is finite and $F=\left\langle E\left(S_{0}\right)\right\rangle \cap G$.

To search for examples of semigroups with a minimum ideal that is completely simple, a natural starting point is completely regular semigroups.

Corollary 8.3 Let $S$ be a completely regular semigroup, with decomposition $S=$ $\bigcup_{\alpha \in \mathcal{Y}} S_{\alpha}$ into a semilattice of completely simple semigroups $S_{\alpha}, \alpha \in \mathcal{Y}$. Then $\omega_{S}^{\ell}$ is finitely generated if and only if $\mathcal{Y}$ has a least element 0 , so that $S_{0}$ forms a minimum ideal that is completely simple, and conditions (1)-(3) of Theorem 8.1 hold.

Proof As $\mathcal{J}$ is a congruence on $S$, Proposition 4.1 tells us that $\omega_{S / \mathcal{J}}^{\ell}$ is finitely generated, and so $\mathcal{Y} \cong S / \mathcal{J}$ has a least element by Corollary 5.7. Conditions (1)-(3) and the converse is immediate from Theorem 8.1.

For the case of bands, Conditions (1)-(3) of Theorem 8.1 reduce noticeably, since maximal subgroups are trivial and $\leq_{\mathcal{L}}$ has a simplified form:

Corollary 8.4 Let $B$ be a band, with decomposition $B=\bigcup_{\alpha \in \mathcal{Y}} B_{\alpha}$ into a semilattice of rectangular bands $B_{\alpha}, \alpha \in \mathcal{Y}$. Then $\omega_{B}^{\ell}$ is finitely generated if and only if the following hold:

(1) $\mathcal{Y}$ has a least element 0 ;

(2) there exists a finite subset $X$ of $B$ such that for every $e \in B$ we have some $x \in X$ with ex $=e$;

(3) there exists an $\mathcal{L}$-class $L$ of $B_{0}$ and a finite subset $W \subseteq L$ such that every element of $L$ is $\rho_{W^{2}}$-related to an element of $W$ via the left congruence $\rho_{W^{2}}$ defined on $B$.

We may use Corollary 8.2 in partnership with Corollaries 8.3 and 8.4 to specialise to completely regular semigroups, and to bands, with minimum ideal having finitely many $\mathcal{R}$-classes.

Corollary 8.5 Let $S$ be a completely regular semigroup, with decomposition $S=$ $\bigcup_{\alpha \in \mathcal{Y}} S_{\alpha}$ into a semilattice of completely simple semigroups $S_{\alpha}, \alpha \in \mathcal{Y}$. Suppose also that $S$ has a minimum ideal $S_{0}$ with finitely many $\mathcal{R}$-classes. Then $\omega_{S}^{\ell}$ is finitely generated if and only if

(1) there exists a finite subset $X$ of $E(S)$ such that for every a $\in S$ we have some $e \in X$ with ae $=a$; 
(2) if $G$ is a maximal subgroup of $S_{0}$, then $G=\langle F \cup V\rangle$ where $V$ is finite and $F=\left\langle E\left(S_{0}\right)\right\rangle \cap G$.

If $S$ is a band, (2) is redundant.

Our current examples of completely regular semigroups with finitely generated universal relation include completely simple semigroups and strong semilattices of groups or rectangular bands (possibly with identity adjoined). In each case they possess a minimum ideal with finitely many $\mathcal{R}$-classes, although we will now construct an example which dictates that this is not a general phenomenon.

The following construction will be used in the desired example, but, as it is quite general, it is convenient to state it separately.

Lemma 8.6 Let $S$ be a semigroup and $U$ a left zero semigroup with $S \cap U=\emptyset$. Suppose that $S$ acts on $U$ on the left via $(s, u) \mapsto s \cdot u$. Let $T=S \cup U$ and define a binary operation $*$ on $T$, extending those on $S$ and $U$, as follows:

$$
s * u=s \cdot u, u * s=u
$$

for all $s \in S, u \in U$. Then $T$ is a semigroup having $U$ as minimum ideal. If $S$ is a monoid with identity 1 and acts monoidally, then $T$ is a monoid with identity 1.

Proof It is clear that every element of $U$ is a left zero for the multiplication. It is easy to check that for any $s, t \in S$ and $u, v \in U$ we have

$$
(s * t) * u=s *(t * u),(s * u) * t=s *(u * t) \text { and }(s * u) * v=s *(u * v)
$$

so that the multiplication is associative. The result follows.

In the next example, by $\mathcal{T}_{U}^{\text {op }}$ we mean the full transformation monoid on $U$ with composition right to left.

Example 8.7 There exists a left regular band monoid with finitely generated universal relation such that the minimum ideal has infinitely many $\mathcal{R}$-classes.

Proof Let $U=\left\{u_{i}: i \in \mathbb{N}^{0}\right\}$ be a left zero semigroup and let $L=\left\{\ell_{i}: i \in \mathbb{N}\right\}$. Define a map $L \times U \rightarrow U$ by $\ell_{i} \cdot u_{0}=u_{0}$ and $\ell_{i} \cdot u_{j}=u_{i}$ for any $i, j \in \mathbb{N}$. This gives us a map from $L$ to $\mathcal{T}_{U}^{\text {op }}$, which can be extended to a morphism from $L^{*}$ to $\mathcal{T}_{U}^{\text {op }}$ and we therefore obtain an action of $L^{*}$ on $U$. Let $M$ be the free left regular band monoid on $L$. Notice that for any $v, w \in L^{*}$ we have

$$
v w v \cdot u_{i}=v w \cdot u_{i} \text { for any } i \in \mathbb{N}^{0},
$$

so that we also have an induced action of $S$ on $U$ given by $\bar{w} \cdot u_{j}=w \cdot u_{j}$, where $\bar{w}$ denotes the natural image of $w \in L^{*}$ in $S$.

Let $T=S \cup U$ be made into a monoid under $*$ as in Lemma 8.6. We claim that $\omega_{T}^{\ell}=\left\langle X^{2}\right\rangle$ where $X=\left\{1, u_{0}, u_{1}\right\}$. To see this we use Corollary 8.4, noting that conditions (1) and (2) are clear by construction. Let $W=\left\{u_{0}, u_{1}\right\}$, and take any $u_{i} \in U \backslash W$. Then $u_{i}=l_{i} u_{1} \rho_{W^{2}} l_{i} u_{0}=u_{0}$, and so Condition (3) holds, thus proving the claim. 
In the case where $\mathcal{H}$ is a congruence on a completely regular semigroup $S$, we may take a different approach to Corollary 8.3. Our aim is to determine the relevant property of the completely simple semigroup in terms of the band $S / \mathcal{H}$ and a maximal subgroup of the minimum ideal of $S$.

Theorem 8.8 Let $S$ be a completely regular semigroup and suppose that $\mathcal{H}$ is a congruence on $S$. Let $S=\bigcup_{\alpha \in \mathcal{Y}} S_{\alpha}$ be the decomposition of $S$ into a semilattice of completely simple semigroups $S_{\alpha}, \alpha \in \mathcal{Y}$. Then $\omega_{S}^{\ell}$ is finitely generated if and only if the following hold:

(1) the universal relation on $S / \mathcal{H}$ is finitely generated;

(2) if $G$ is a maximal subgroup of $S_{0}$, where 0 is the least element of $\mathcal{Y}$ (the existence of 0 follows from (1)), then $G=\langle F \cup V\rangle$ where $V$ is finite and $F=\left\langle E\left(C_{0}\right)\right\rangle \cap G$ where $C_{0}$ is the union of finitely many $\mathcal{R}$-classes of $S_{0}$.

Proof We first suppose that $\omega_{S}^{\ell}=\left\langle U^{2}\right\rangle$ for a finite set $U \subseteq S$. As $\mathcal{H}$ is a congruences on $S$, Proposition 4.1 tells us that the universal relation on the quotient $S / \mathcal{H}$ is also finitely generated. Condition (2) follows from Corollary 8.3.

Conversely, suppose that (1) and (2) hold. Let $E$ be a finite subset of $E(S)$ such that the universal relation on $S / \mathcal{H}$ is finitely generated by $[E] \times[E]$ where $[E]=\left\{H_{f}\right.$ : $f \in E\}$. As in the proof of Theorem 8.1 we may assume that $G$ is an $\mathcal{H}$-class of $S_{0}$ and there exists a finite subset $W$ in $S$ such that for any $g, h \in G$ we have $g \rho_{W^{2}} h$.

Let $X=E \cup W \cup\{e\} \cup E e$, where $e$ is the identity of $G$. We claim that $\omega_{S}^{\ell}=\left\langle X^{2}\right\rangle$. Let $a \in S$. Then $[a] \leq \mathcal{L}[f]$ for some $f \in E$ and so $a \leq_{\mathcal{L}} f$. Now

$$
a=a f \rho_{X^{2}} a e=b \mathcal{L} e
$$

and $e b \in G$, so that $e b \rho_{W^{2}} e$. If $g=g^{2} \mathcal{H} b$, then $b \rho_{W^{2}} g e=g$ and as $\rho_{W^{2}} \subseteq \rho_{X^{2}}$, so $b \rho_{X^{2}} g$. It remains to show that $g \rho_{X^{2}} e$.

As $[g] \rho_{[E]^{2}}[e]$, there exists a sequence

$$
[g]=\left[t_{1}\right]\left[c_{1}\right],\left[t_{1}\right]\left[d_{1}\right]=\left[t_{2}\right]\left[c_{2}\right], \ldots,\left[t_{n}\right]\left[d_{n}\right]=[e],
$$

for $\left[t_{i}\right] \in(S / \mathcal{H})^{1}$ and $\left(\left[c_{i}\right],\left[d_{i}\right]\right) \in[E]^{2}, 1 \leq i \leq n$. Now

$$
g \mathcal{H} t_{1} c_{1}, t_{1} d_{1} \mathcal{H} t_{2} c_{2}, \ldots, t_{n} d_{n} \mathcal{H} e
$$

Multiplying by $e$ on the right we have

$$
g \mathcal{H} t_{1}\left(c_{1} e\right), t_{1}\left(d_{1} e\right) \mathcal{H} t_{2}\left(c_{2} e\right) \ldots, t_{n}\left(d_{n} e\right) \mathcal{H} e
$$

Now suppose $p, q \in L_{e}$ where $p \mathcal{H} q \mathcal{H} r=r^{2}$. Then ep $\mathcal{H}$ eq $\mathcal{H}$ e and so ep $\rho_{W^{2}}$ eq. This implies rep $\rho_{W^{2}}$ req and hence $p \rho_{W^{2}} q$. Thus

$$
g \rho_{W^{2}} t_{1}\left(c_{1} e\right) \rho_{X^{2}} t_{1}\left(d_{1} e\right) \rho_{W^{2}} t_{2}\left(c_{2} e\right) \ldots \rho_{W^{2}} e,
$$

and so $g \rho_{X^{2}} e$ and $\omega_{S}^{\ell}=\left\langle X^{2}\right\rangle$, as required. 
Remark 8.9 All the results in this section may be easily adapted to the pseudo-finite case. For example, in Theorem 8.1 the pseudo-finiteness of $S$ guarantees that the lengths of the $(F \cup V)^{2}$-sequences and of the $W^{2}$-sequences in (2) and (3) respectively may be bounded, with the converse also clearly holding.

Open Question 8.10 Every pseudo-finite semigroup considered in this article has the property that it contains an ideal which is completely simple. The contrast to the weaker case where $\omega^{l}$ is finitely generated is highlighted by Theorem 5.1 and Proposition 5.3. In view of this we ask whether all pseudo-finite semigroups have this property? By the work of Sect. 8, a positive answer to this question would give a complete description of all pseudo-finite semigroups.

Acknowledgements The authors are grateful to Professor Garth Dales and Dr. Jared White for asking the question that led to the investigation in this article.

Open Access This article is distributed under the terms of the Creative Commons Attribution 4.0 International License (http://creativecommons.org/licenses/by/4.0/), which permits unrestricted use, distribution, and reproduction in any medium, provided you give appropriate credit to the original author(s) and the source, provide a link to the Creative Commons license, and indicate if changes were made.

\section{References}

1. Anick, D.J.: On the homology of associative algebras. Trans. Am. Math. Soc. 296, 641-659 (1986)

2. Bieri, R.: Homological dimension of discrete groups. Mathematics Department, Queen Mary College, London, 1976. Queen Mary College Mathematics Notes

3. Clifford, A.H., Preston, G.B.: The algebraic theory of semigroups, vol. II, American Mathematical Society, Providence, pp 140-141 (1967)

4. Cohen, D.E.: A monoid which is right $\mathrm{FP}_{\infty}$ but not left $\mathrm{FP}_{1}$. Bull. London Math. Soc. 24, 340-342 (1992)

5. Cohen, D.E.: String rewriting and homology of monoids. Math. Struct. Comput. Sci. 7, 207-240 (1997)

6. Dales, G., White, J.: Private communication (2016)

7. Gould, V.: A notion of rank for right congruences on semigroups. Comm. Algebra 33, 4631-4656 (2005)

8. Gould, V., Hartmann, M.: Coherency, free inverse monoids and related free algebras. Math. Proc. Cambdridge Phil. Soc. 163, 23-45 (2017)

9. Gray, R., Pride, S.: Homological finiteness properties of monoids, their ideals and maximal subgroups. J.P.A.A 215, 3005-3024 (2011)

10. Guba, V., Pride, S.: On the left and right cohomological dimension of monoids. Bull. London Math. Soc. 30, 391-396 (1998)

11. Hotzel, E.: On semigroups with maximal conditions. Semigroup Forum 11, 337-362 (1975)

12. Howie, J.M.: Idempotents in completely 0-simple semigroups. Glasgow Math. J. 19, 109-113 (1978)

13. Howie, J.M.: Fundamentals of Semigroup Theory. Oxford University Press, Oxford (1995)

14. Kilp, M., Knauer, U., Mikhalev, A.V.: Monoids, Acts, and Categories. de Gruyter, Berlin (2000)

15. Kobayashi, Y.: The homological finiteness property $F P_{1}$ and finite generation of monoids. Int. J. Algebra Comput. 17, 593-605 (2007)

16. Kobayashi, Y.: The homological finiteness properties left-, right- and bi- $F P_{n}$ of monoids. Commun. Algebra 38, 3975-3986 (2010)

17. Kozhukhov, I.: On semigroups with minimal or maximal condition on left congruences. Semigroup Forum 21, 337-350 (1980)

18. McGlashan, S., Pasku, E., Pride, S.J.: Finiteness conditions for rewriting sytems. I.J.A.C 15, 175-205 (2005)

19. Miller, C., Ruškuc, N.: Right noetherian semigroups. arXiv:1811.08408 
20. Normak, P.: On noetherian and finitely presented $M$-sets. Uč Zap. Tartu Gos. Univ. 431, 37-46 (1977). (in Russian)

21. Pride, S.: Homological finiteness conditions for groups, monoids, and algebras. Commun. Algebra 34, 3525-3536 (2006)

22. Pride, S., Otto, F.: For rewriting systems the topological finiteness conditions FDT and FHT are not equivalent. J. London Math. Soc. 69, 363-382 (2004)

23. Ruškuc, N.: On large subsemigroups and finiteness conditions of semigroups. Proc. London Math. Soc. 76, 383-405 (1998)

24. White, J.T.: Finitely-generated left ideals in banach algebra on groups and semigroups. Studia Mathematica 239, 67-99 (2017)

Publisher's Note Springer Nature remains neutral with regard to jurisdictional claims in published maps and institutional affiliations.

\section{Affiliations}

\section{Yang Dandan ${ }^{1}$. Victoria Gould ${ }^{2}$ (1) - Thomas Quinn-Gregson ${ }^{3}$. Rida-E Zenab ${ }^{2,4}$}

$凶$ Victoria Gould

victoria.gould@york.ac.uk

Yang Dandan

ddyang@xidian.edu.cn

Thomas Quinn-Gregson

thomas.quinn-gregson@tu-dresden.de

Rida-E Zenab

ridaezenab@iba-suk.edu.pk; rzz500@york.ac.uk

1 School of Mathematics and Statistics, Xidian University, Xi' an 710071, People's Republic of China

2 Department of Mathematics, University of York, Heslington, York YO10 5DD, UK

3 Institut für Algebra, Technische Universität Dresden, 01062 Dresden, Germany

4 Department of Mathematics, Sukkur IBA University, Sukkur, Pakistan 